\title{
Hippocampal connectivity with retrosplenial cortex drives neocortical tau accumulation and memory function
}

Jacob Ziontz ( $\sim$ jacob_ziontz@berkeley.edu )

University of California, Berkeley https://orcid.org/0000-0002-1995-3827

Jenna Adams

University of California, Berkeley https://orcid.org/0000-0002-6702-3851

Theresa Harrison

University of California, Berkeley

Suzanne Baker

Lawrence Berkeley National Laboratory https://orcid.org/0000-0003-0209-3127

William Jagust

University of California, Berkeley https://orcid.org/0000-0002-4458-113X

\section{Article}

Keywords: Alzheimer's disease, Tau Pathology, Cognitive Decline, Neural Connectivity, Episodic Memory, Aging

Posted Date: February 23rd, 2021

DOl: https://doi.org/10.21203/rs.3.rs-235539/v1

License: (9) (1) This work is licensed under a Creative Commons Attribution 4.0 International License. Read Full License 
Hippocampal connectivity with retrosplenial cortex drives neocortical tau

\section{accumulation and memory function}

4 Authors: Jacob Ziontz ${ }^{1 *}$, Jenna N. Adams. ${ }^{1}$, Teresa M. Harrison. ${ }^{1}$, Suzanne L. Baker. ${ }^{2}$, William

5 J. Jagust ${ }^{1,2}$.

6 Affiliations:

$7 \quad{ }^{1}$ Helen Wills Neuroscience Institute, UC Berkeley, Berkeley, United States

$8{ }^{2}$ Molecular Biophysics and Integrated Bioimaging, Lawrence Berkeley National Laboratory,

9 Berkeley, United States

10 *Correspondence: Jacob Ziontz, jacob_ziontz@berkeley.edu

11

12

13

14

15

16

17

18

19

20

21

22 
1 Abstract: The mechanisms underlying accumulation of Alzheimer's disease (AD)-related tau

2 pathology outside of the medial temporal lobe (MTL) in older adults are unknown but crucial to

3 understanding cognitive decline. Neural connectivity has recently been implicated in the

4 propagation of tau in humans, consistent with data from animal studies. Using resting state

5 functional connectivity and tau PET imaging, we examined MTL structures involved in medial

6 parietal tau deposition in cognitively normal older adults. Functional connectivity between

7 retrosplenial cortex and hippocampus, but not entorhinal cortex, correlated with tau in medial

8 parietal lobe. Further, hippocampal-retrosplenial connectivity strength modulated the correlation

9 between MTL and medial parietal lobe tau, as well as between medial parietal tau and episodic

10 memory. Medial parietal tau spread thus reflects patterns of neural connectivity that represent a

11 critical step in the evolution of cognitive dysfunction in aging and AD.

\section{Introduction}

14 The microtubule-associated protein tau forms neurofibrillary tangles (NFTs) in its

15 hyperphosphorylated state, which together with amyloid- $\beta(\mathrm{A} \beta)$ plaques are the hallmark

16 neuropathologies of Alzheimer's disease (AD). Early histopathology studies of these NFTs

17 described tau pathology as first appearing in medial temporal lobe (MTL) structures such as the

18 entorhinal cortex and hippocampus before later accumulating in limbic areas and association

19 cortex ${ }^{1}$. In recent years, the use of positron emission tomography (PET) imaging has allowed the

20 in vivo characterization of the distribution and extent of pathological tau burden in patients with

$21 \mathrm{AD}$ as well as cognitively healthy older adults ${ }^{2,3}$. A developing body of work has used other

22 neuroimaging modalities in concert with PET to investigate what factors and characteristics of

23 the aging brain lead to stereotypical patterns of tau pathology. 
Though the mechanisms underlying patterns of tau spread are not yet fully understood,

2 there is converging evidence of transsynaptic propagation of tau through coactive neurons.

3 Studies in vitro and in animal models suggest that tau pathology can be transferred between

4 synaptic connections, and that enhanced neuronal activity stimulates the release of pathological

5 tau and increases downstream accumulation ${ }^{4-6}$. In humans, transneuronal tau spread has been

6 investigated by comparing the topography of tau accumulation with measures of structural and

7 functional connectivity ${ }^{7-13}$. Graph theoretic measures of resting state functional magnetic

8 resonance imaging ( $\mathrm{fMRI}$ ) indicate that connected nodes in the brain tend to exhibit greater tau

9 accumulation ${ }^{7}$, and computational modeling of tau spread based on both structural and

10 functional connectivity is highly predictive of the observed pattern of tau accumulation ${ }^{8}$.

11 Further, the degree of between-region functional connectivity is associated with the covariance

12 of change in tau PET signal over time ${ }^{9}$. Given that the degree of connectivity across the cortex

13 has been shown to relate to the pattern of tau accumulation, connectivity measures in brain

14 regions that first exhibit tau pathology may provide insights into the structures and processes

15 involved in the spread of tau in aging and AD.

16 NFTs are thought to originate in the transentorhinal region, an area spanning the lateral

17 entorhinal cortex and medial perirhinal cortex in humans ${ }^{14,15}$. Tau pathology is in fact observed

18 in MTL structures such as the entorhinal cortex and hippocampus in virtually all older adults ${ }^{16}$.

19 However, its accumulation in cortical areas outside of the MTL, perhaps facilitated by A $\beta^{17}$, is

20 often a feature of the earliest stages of $\mathrm{AD}$, with medial parietal cortex being a particularly

21 common area of deposition ${ }^{18,19}$. A recent study from our group found that functional

22 connectivity of the anterolateral entorhinal cortex (alEC), the entorhinal area most proximal to

23 the transentorhinal region, showed the strongest association with neocortical tau PET signal in 
1 cognitively normal older adults ${ }^{11}$. Tau may thus initially propagate directly to some neocortical

2 areas via connectivity with the alEC. However, cortical regions such as the medial parietal area

3 that have not been reported to have strong structural connectivity to alEC ${ }^{20,21}$ also develop tau

4 pathology as AD progresses. For these regions, it is plausible that connections with other MTL

5 structures that show later tau deposition, including the posteromedial entorhinal cortex (pmEC)

6 and hippocampus, underly tau spread from MTL to neocortex.

7 The medial parietal lobe is notable for its characteristic glucose hypometabolism in early

$8 \mathrm{AD}^{22}$, and exhibits greater tau accumulation relative to surrounding cortical areas both in

9 patients with $\mathrm{AD}$ and cognitively healthy older individuals ${ }^{19,23}$. This region also comprises a

10 large part of the posterior medial (PM) memory network, which together with the anterior

11 temporal (AT) memory network represents a framework of two distinct large-scale neocortical

12 memory networks with separable anatomical and functional connectivity with the MTL and

13 support of different aspects of memory and cognitive function ${ }^{24,25}$. Though greater tau

14 accumulation in regions of the AT network compared to PM regions is typically observed in

15 cognitively normal older adults, there is a substantial increase in PM tau in mild cognitive

16 impairment (MCI) and $\mathrm{AD}$ individuals ${ }^{18}$. Tau accumulation in medial parietal lobe thus

17 represents spread into the PM network, which may be a key condition under which memory

18 performance begins to worsen prior to the clinical presentation of disease. Examining the role of

19 connectivity in tau's pathway to the medial parietal lobe may help explain why this area is such a

20 specific target of AD pathology and what effect the spread of tau has on memory processing.

21 In the present study, we use functional connectivity from resting state fMRI to investigate

22 how tau spreads from the MTL to the medial parietal lobe. In a sample of cognitively normal

23 older adults, we measured functional connectivity between key MTL subregions (alEC, pmEC 
1 and hippocampus) and medial parietal lobe, and tested whether this connectivity was related to

2 tau deposition in medial partial lobe. Given that hippocampus is known to develop tau pathology

3 after entorhinal cortex and exhibits strong structural connectivity with medial parietal lobe

4 regions ${ }^{26}$, we hypothesized that the degree of functional connectivity with hippocampus would

5 better predict tau burden in medial parietal lobe than functional connectivity with either

6 entorhinal subregion. In addition, because pmEC also demonstrates some connectivity with

7 medial parietal areas ${ }^{21}$ and alEC is the earliest region of tau accumulation, we tested whether

8 these regions would also contribute to medial parietal tau. Further, we were interested in

9 examining how connectivity between the MTL and medial parietal lobe was related to both the

10 correspondence of tau between the two regions and to cognitive function. We hypothesized that

11 MTL and medial parietal tau would be more correlated as functional connectivity between these

12 regions increased, and that greater tau burden and functional connectivity together would be

13 associated with worse memory performance.

\section{Results}

15 Participants

16 We analyzed data from 97 cognitively unimpaired older adults from the Berkeley Aging

17 Cohort Study (BACS) with structural and resting state functional 3T MRI scans. These

18 individuals were also administered concurrent tau PET scans with ${ }^{18} \mathrm{~F}$-Flortaucipir (FTP) and A $\beta$

19 PET scans with ${ }^{11} \mathrm{C}$-Pittsburgh Compound $\mathrm{B}(\mathrm{PiB})$, as well as a standard cognitive assessment.

20 Demographic information for all participants is shown in Table 1.

Table 1. Participant characteristics. Demographic information for sample of 97 cognitively normal older adults including age, years of education, mini mental state examination (MMSE) score, global beta amyloid positron emission tomography (A $\beta$ PET) signal, sex, beta amyloid positivity status $(A \beta+)$, and apolipoprotein E positivity status $(\varepsilon 4+)$. 


\begin{tabular}{|c|c|c|}
\hline & $\begin{array}{l}\text { Mean (SD) or } \mathrm{n} \\
(\%)\end{array}$ & Range \\
\hline Age (years) & $76.4(6.1)$ & $60-93$ \\
\hline $\begin{array}{l}\text { Education } \\
\text { (years) }\end{array}$ & $16.8(1.9)$ & $12-20$ \\
\hline MMSE & $28.6(1.3)$ & $25-30$ \\
\hline Global A PET & $1.17(0.25)$ & $0.92-1.89$ \\
\hline Sex (female) & $58(59.8)$ & \\
\hline$A \beta+$ & $43(45.7)$ & \\
\hline$A P O E \varepsilon 4+$ & $28(29.8)$ & \\
\hline
\end{tabular}

\section{Hippocampus exhibits strong resting state functional connectivity with retrosplenial cortex}

We first investigated resting state functional connectivity between MTL and medial parietal lobe in our sample. We selected three regions of interest within MTL: the anterolateral

(alEC) and posteromedial (pmEC) entorhinal cortices as well as the hippocampus (Figure 1a).

Within the medial parietal lobe, we identified the retrosplenial cortex as a region where tau is

thought to spread to the neocortex via the MTL ${ }^{15}$ and with known structural and functional connectivity with MTL 21,24,26. Hippocampus and retrosplenial cortex (“isthmus cingulate”, see

Methods) regions of interest were derived using the FreeSurfer segmentation of each

participant's native space MRI, and template space alEC and pmEC were defined from a

previous high-resolution MRI study ${ }^{27}$. We performed bilateral region-to-region functional connectivity analyses with semipartial correlations adjusting for age and sex.

Next, we carried out one-sample t-tests using $\beta$-weights from region-to-region semipartial correlations to see if functional connectivity between each region was significantly different

from 0 (Figure 1b). We found significant connectivity between hippocampus and retrosplenial cortex $(\beta=0.45, p<0.001)$, as well as between pmEC and retrosplenial cortex $(\beta=0.07, p=$

6 0.007). We further compared the connectivity of these two pathways using a paired samples t17 test and found that hippocampal-retrosplenial (HC-RsC) connectivity was significantly greater 
1 than pmEC-RsC connectivity $(t(96)=15.74, p<0.001)$. By contrast, no significant connectivity was observed between alEC and retrosplenial cortex $(\beta=0.00, p=0.891)$. Thus, hippocampus

3 and to a lesser extent pmEC, but not alEC, exhibited resting state functional connectivity with 4 retrosplenial cortex in our sample of cognitively normal older adults.

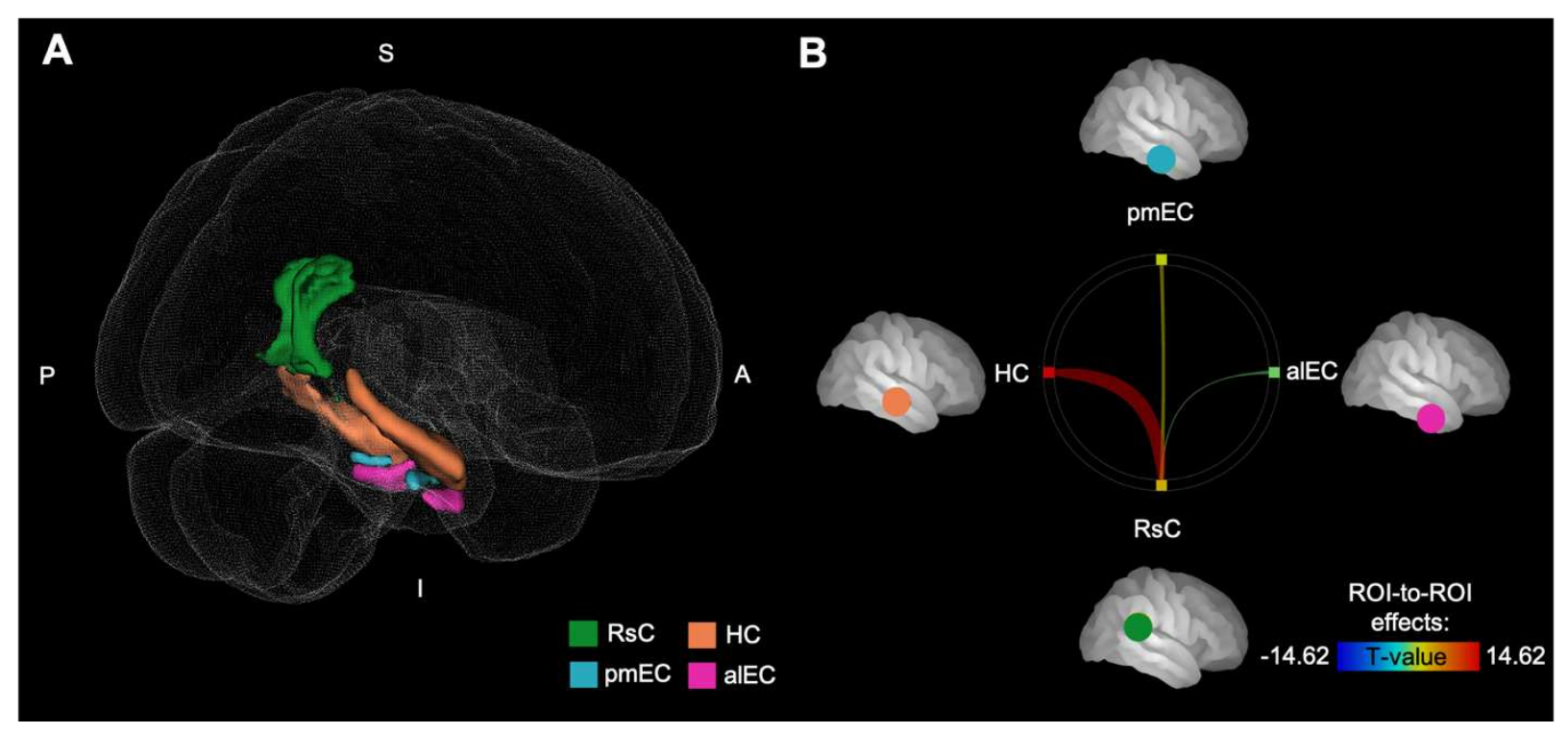

Figure 1. Hippocampus exhibits strong resting state functional connectivity with retrosplenial cortex. (A) Regions of interest for functional connectivity analysis $(n=97)$. Anterolateral entorhinal cortex (alEC), posteromedial entorhinal cortex (pmEC), and hippocampus (HC) were included from the medial temporal lobe, as well as retrosplenial cortex (RsC). (B) Retrosplenial cortex exhibits functional connectivity with hippocampus $(\beta=0.45, p<0.001)$ and posteromedial entorhinal cortex $(\beta=0.07, p=0.007)$, but not anterolateral entorhinal cortex $(\beta=0.00, p=0.89)$. Line color and thickness correspond to the Tstatistic of semipartial correlations of resting state activity between regions.

7 Hippocampal-retrosplenial connectivity strength is related to medial parietal tau pathology Having observed strongest functional connectivity between hippocampus and

9 retrosplenial cortex, we next sought to investigate the extent to which this connectivity is

10 associated with tau accumulation in medial parietal lobe. We operationalized connectivity

11 strength by again extracting the $\beta$-weights from region-to-region semipartial correlations for each

12 participant. Tau pathology was quantified as the proportion of voxels above an a priori threshold 
1 for tau PET (SUVR > 1.4), following previous work that demonstrated this to be a reliable

2 marker of AD-related tau pathology ${ }^{23}$. We computed this suprathreshold tau in a medial parietal

3 lobe composite region comprising the retrosplenial cortex, precuneus, and posterior cingulate

4 cortex such that for each participant, the number of voxels above threshold was divided by the

5 total number of voxels in this region. To visualize this tau signal, we also computed the

6 proportion of participants above threshold in each voxel of the composite region. (Figure 2a).

7 Using multivariate linear regression, we then examined the association of suprathreshold

8 medial parietal lobe tau with connectivity strength between each MTL subregion and

9 retrosplenial cortex. Adjusting for age, sex, and global A $\beta$ PET signal, we found that HC-RsC

10 connectivity strength was associated with suprathreshold tau in the medial parietal lobe $(\beta=$

$110.145, p=0.004 ;$ Figure $2 \mathrm{~b}$ ). The global $\mathrm{A} \beta$ term from this model was also associated with

12 suprathreshold medial parietal tau $(\beta=0.113, p=0.014)$. In contrast with HC-RsC, linear

13 regression models adjusting for age, sex, and global $\mathrm{A} \beta$ revealed that neither alEC-RsC $(\beta=$

$140.120, p=0.145)$ nor pmEC-RsC connectivity strength $(\beta=0.015, p=0.842)$ were associated

15 with medial parietal lobe tau (Figure 2c-d). In a separate model, we further examined whether

16 individuals with more global $\mathrm{A} \beta$ exhibited a stronger relationship between $\mathrm{HC}-\mathrm{RsC}$ and medial

17 parietal tau. Adjusting for age and sex, we did not observe a significant interaction between HC

18 RsC connectivity and global $\mathrm{A} \beta(\beta=0.442, p=0.141)$. 


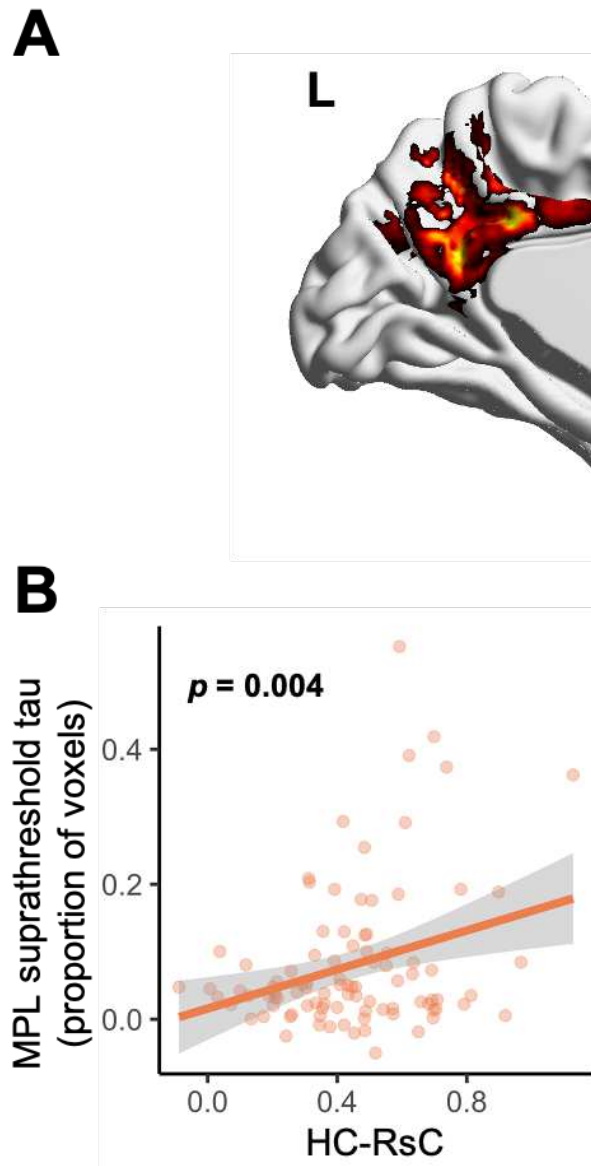

1 between regions.

\section{Voxels $>1.4$}

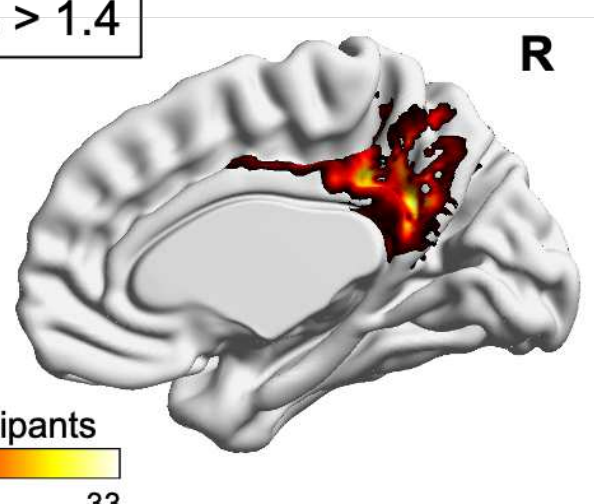

3

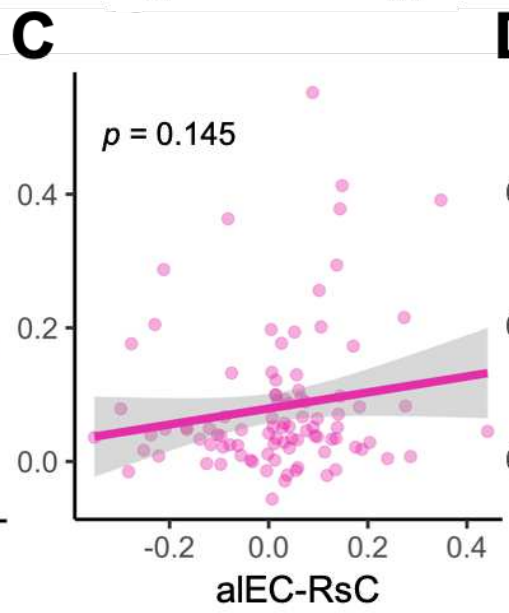

D

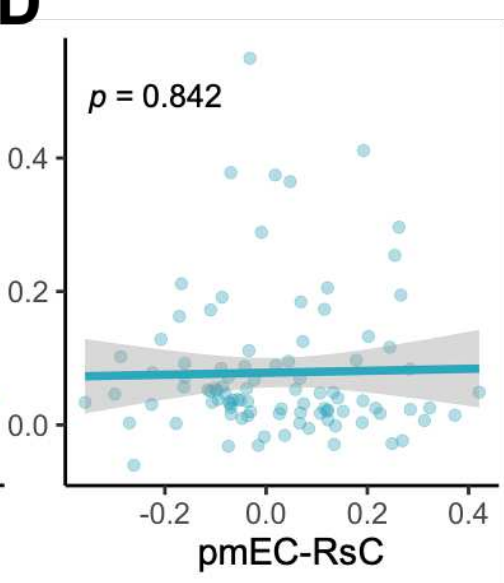

FC strength $(\beta)$

Figure 2. Hippocampal-retrosplenial connectivity strength is related to medial parietal tau pathology. (A) Percent of participants above tau threshold (FTP SUVR > 1.4) for each voxel in medial parietal lobe (MPL) region comprising retrosplenial cortex, precuneus, and posterior cingulate cortex. (B) Adjusting for age, sex, and global beta amyloid (A $\beta$ ), hippocampal-retrosplenial (HC-RsC) resting state functional connectivity strength is associated with mean suprathreshold tau within MPL. MPL suprathreshold tau defined as proportion of voxels above threshold (FTP SUVR > 1.4) within composite region. (C) Anterolateral entorhinal cortex-retrosplenial (alEC-RsC) and (D) posteromedial entorhinal cortexretrosplenial ( $\mathrm{pmEC}-\mathrm{RsC}$ ) connectivity strength are not associated with MPL tau. Significance value for each plot corresponds to effect of each term from linear regression model. Functional connectivity (FC) strength quantified as $\beta$-values extracted from semipartial correlations

was specific to retrosplenial cortex and not a general effect of strong resting state functional

connectivity, we identified a control region within the superior frontal gyrus (SFG) analogous to 
1 the medial portion of Brodmann area 10 from the Brainnetome Atlas ${ }^{28}$. Like retrosplenial cortex,

2 this region is known to be part of the default mode network but does not have extensive

3 structural connections with hippocampus and exhibited low signal dropout in our sample. Similar

4 to retrosplenial cortex, we observed significant resting state functional connectivity between

5 hippocampus and SFG $(\beta=0.40, p<0.001)$, though not between SFG and alEC $(\beta=0.02, p=$

$60.439)$ or $\operatorname{pmEC}(\beta=-0.02, p=0.379$; Supplementary Figure 1a). However, in contrast with

7 retrosplenial cortex, we did not observe an association between the strength of hippocampus-

8 SFG connectivity and medial parietal lobe tau $(\beta=0.006, p=0.907$; Supplementary Figure $1 \mathrm{~b})$.

9 We further wanted to verify that connectivity between hippocampus and retrosplenial

10 cortex was associated specifically with tau in medial parietal lobe and not also in other early tau-

11 accumulating regions. To this end, we examined tau within entorhinal cortex and inferior

12 temporal cortex, a region often used as a marker for early tau accumulation in aging ${ }^{29,30}$. Again

13 using linear regression adjusting for age, sex, and global $\mathrm{A} \beta$, we found that $\mathrm{HC}$-RsC connectivity

14 was not associated with suprathreshold tau in the entorhinal cortex $(\beta=-0.017, p=0.843$;

15 Supplementary Figure 2a). Notably, though suprathreshold tau signal in medial parietal lobe and

16 inferior temporal cortex were highly correlated $(r=0.796, p<0.001)$, HC-RsC was only

17 associated at trend level with inferior temporal tau $(\beta=0.150, p=0.082$; Supplementary Figure

18 2b). Finally, to confirm that the relationship between connectivity and pathology was specific to

19 tau, we examined the association between $\mathrm{HC}-\mathrm{RsC}$ and $\mathrm{A} \beta$ burden in the medial parietal lobe.

20 Adjusting for age and sex, $\mathrm{HC}-\mathrm{RsC}$ connectivity was not associated with medial parietal lobe $\mathrm{A} \beta$

21 PET signal $(\beta=0.172, p=0.193$; Supplementary Figure $2 c)$. Taken together, these results

22 demonstrate the specificity of tau pathology accumulation in medial parietal lobe via direct

23 connectivity with hippocampus in cognitively unimpaired older adults. 
1 Hippocampal-retrosplenial connectivity modulates the relationship between medial temporal

2 and medial parietal tau

3 To further examine the role of functional connectivity in the spread of tau pathology from

4 MTL, we tested whether HC-RsC connectivity strength modulated how closely MTL tau

5 corresponded with medial parietal lobe tau. Measuring tau in MTL, particularly in hippocampus,

6 is challenging given the confound of signal contamination from off-target FTP binding in

7 choroid plexus. To address this, we quantified MTL tau pathology with hippocampal FTP SUVR

8 using Rousset geometric transfer matrix partial volume correction, which minimizes choroid

9 plexus spillover ${ }^{31}$. As an additional precaution, we adjusted for choroid plexus FTP signal in our

10 linear regression model, in addition to age, sex, and global $\mathrm{A} \beta$ (Table 2).

11 As expected, there was a significant main effect of hippocampal tau $(\beta=0.222, p=$

$120.003)$ as well as a main effect of HC-RsC $(\beta=0.120, p=0.007)$ on medial parietal lobe

13 suprathreshold tau. Critically, we also observed an interaction between hippocampal tau and HC-

$14 \mathrm{RsC}$ connectivity ( $\beta=0.635, p=0.006$; Figure 3$)$ such that there was a stronger association

15 between MTL and medial parietal lobe tau with greater functional connectivity between these

16 regions. To further verify that choroid plexus spillover into hippocampus was not driving these

17 results, we replicated this analysis using partial volume corrected entorhinal tau PET, and found

18 a trend-level interaction between entorhinal tau and HC-RsC in predicting medial parietal tau ( $\beta$

$19=0.265, p=0.069$; Supplementary Figure 3). These findings implicate functional connectivity as

20 a crucial factor in the spread of tau from hippocampus to medial parietal lobe. 
Table 2. Model results. Examined variables from linear regression model examining the relationship between medial parietal lobe (MPL) suprathreshold tau and age, sex, global beta amyloid (A $\beta$ ), choroid plexus signal (partial volume corrected FTP SUVR), hippocampus mean tau (partial volume corrected FTP SUVR), hippocampal-retrosplenial (HC-RsC) functional connectivity (FC) strength, and hippocampus mean tau x HC-RsC FC strength interaction. Bolded term indicates interaction effect visualized in Figure 3.

\begin{tabular}{|l|l|}
\hline Independent variables: & $\begin{array}{l}\text { Dependent variable: } \\
\text { MPL suprathreshold tau }\end{array}$ \\
\hline age & $\beta=-0.001$ \\
& $p=0.375$ \\
\hline sex & $\beta=0.013$ \\
& $p=0.508$ \\
\hline global A $\beta$ & $\beta=0.052$ \\
& $p=0.229$ \\
\hline choroid plexus signal & $\beta=0.016$ \\
& $p=0.746$ \\
\hline hippocampus mean tau & $\beta=0.222$ \\
& $p=0.003$ \\
\hline HC-RsC FC strength & $\beta=0.120$ \\
\hline hippocampus mean tau $\mathbf{x}$ HC-RsC FC & $\boldsymbol{\beta}=0.007$ \\
strength & $\mathbf{p}=\mathbf{0 . 0 0 6}$ \\
\hline Constant & $\beta=-0.009$ \\
& $\mathrm{p}=0.917$ \\
\hline
\end{tabular}




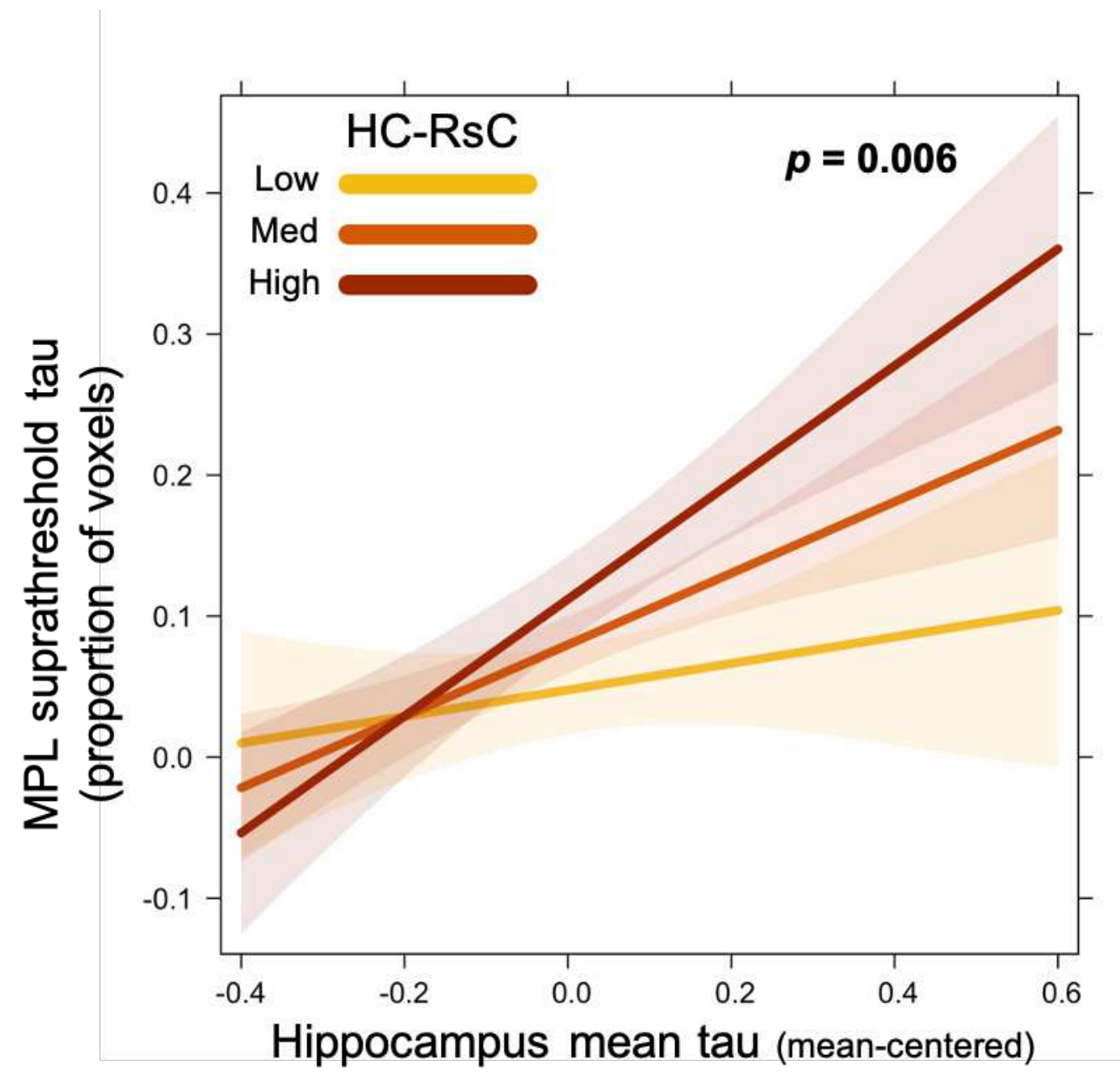

Figure 3. Hippocampal-retrosplenial connectivity modulates the relationship between medial temporal and medial parietal tau.

Visualization of interaction between hippocampus mean tau (partial volume corrected FTP SUVR) and hippocampal-retrosplenial functional connectivity strength (HC-RsC) from linear regression model (see Table 2). Medial parietal lobe (MPL) suprathreshold tau is associated with a hippocampal tau $\mathrm{x}$ HC-RsC interaction. Plot displays the relationship predicted by linear regression at low $\left(10^{\text {th }}\right.$ percentile $)$, median, and high $\left(90^{\text {th }}\right.$ percentile $) \mathrm{HC}-\mathrm{RsC}$.

Hippocampal-retrosplenial connectivity modulates the relationship between medial temporal and medial parietal tau Having observed that increased functional connectivity between hippocampus and

6 retrosplenial cortex modulates tau pathology burden in medial parietal lobe, we sought to test if

7 connectivity and tau in these areas might also interact to predict cognitive function. We 
1 constructed a composite episodic memory measure consisting of the mean z-score of four

2 episodic memory tasks: the California Verbal Learning Test (CVLT) immediate free recall,

3 CVLT long-delay free recall, Visual Reproduction (VR) immediate recall, and VR delay recall

$4 \quad{ }^{32}$. Adjusting for age, years of education, practice effects, and global $A \beta$, we did not observe a

5 significant main effect of either medial parietal lobe tau $(\beta=1.111, p=0.318)$ or HC-RsC

6 connectivity $(\beta=0.073, p=0.861)$ on episodic memory. However, we did find a significant

7 interaction between medial parietal tau and hippocampal-retrosplenial connectivity $(\beta=-9.482, p$

$8=0.018$ ), such that episodic memory performance was poorest when both medial parietal lobe

9 tau and hippocampal-retrosplenial connectivity were greatest (Table 3). To further examine

10 which subdomain of episodic memory might drive this finding, we broke down the episodic

11 memory composite score into a verbal memory component consisting of the two CVLT tasks,

12 and a visuospatial memory component consisting of the two visual reproduction tasks. The

13 interaction between medial parietal lobe tau and hippocampal-retrosplenial connectivity was

14 associated with visuospatial memory performance $(\beta=-12.016, p=0.006$; Figure $4 \mathrm{a})$, but not

15 verbal memory performance $(\beta=-6.933, p=0.145$; Figure $4 \mathrm{~b})$. Taken together, these findings

16 indicate that the combination of greater tau in medial parietal lobe and greater connectivity

17 between hippocampus and retrosplenial cortex is associated with poorer episodic memory, an

18 effect that is perhaps driven by a relationship with visuospatial memory in particular.

Table 3. Model results. Examined variables from linear regression model examining the relationship between episodic memory performance (California Verbal Learning Test immediate and long-delay free recall, Visual Reproduction immediate and delay recall) and age, years of education, practice effects (see Methods), global beta amyloid (A $\beta$ ), medial parietal lobe (MPL) suprathreshold tau, hippocampal-retrosplenial (HC-RsC) functional connectivity (FC) strength, and MPL suprathreshold tau x HC-RsC FC strength interaction. 


\begin{tabular}{|l|l|}
\hline Independent variables: & $\begin{array}{l}\text { Dependent variable: } \\
\text { Episodic memory } \\
\text { composite }\end{array}$ \\
\hline age & $\begin{array}{l}\beta=-0.046 \\
\mathrm{p}=0.006\end{array}$ \\
\hline years education & $\beta=0.013$ \\
& $\mathrm{p}=0.508$ \\
\hline practice effects & $\beta=0.234$ \\
& $\mathrm{p}=0.029$ \\
\hline global A $\beta$ & $\beta=-0.256$ \\
& $\mathrm{p}=0.475$ \\
\hline MPL suprathreshold tau & $\beta=1.113$ \\
& $\mathrm{p}=0.288$ \\
\hline HC-RsC FC strength & $\beta=0.071$ \\
& $\mathrm{p}=0.857$ \\
\hline MPL suprathreshold tau $\mathbf{x}$ HC-RsC & $\boldsymbol{\beta}=\mathbf{- 9 . 4 8 2}$ \\
FC strength & $\mathbf{p}=\mathbf{0 . 0 1 8}$ \\
\hline Constant & $\beta=0.048$ \\
& $\mathrm{p}=0.917$ \\
\hline
\end{tabular}
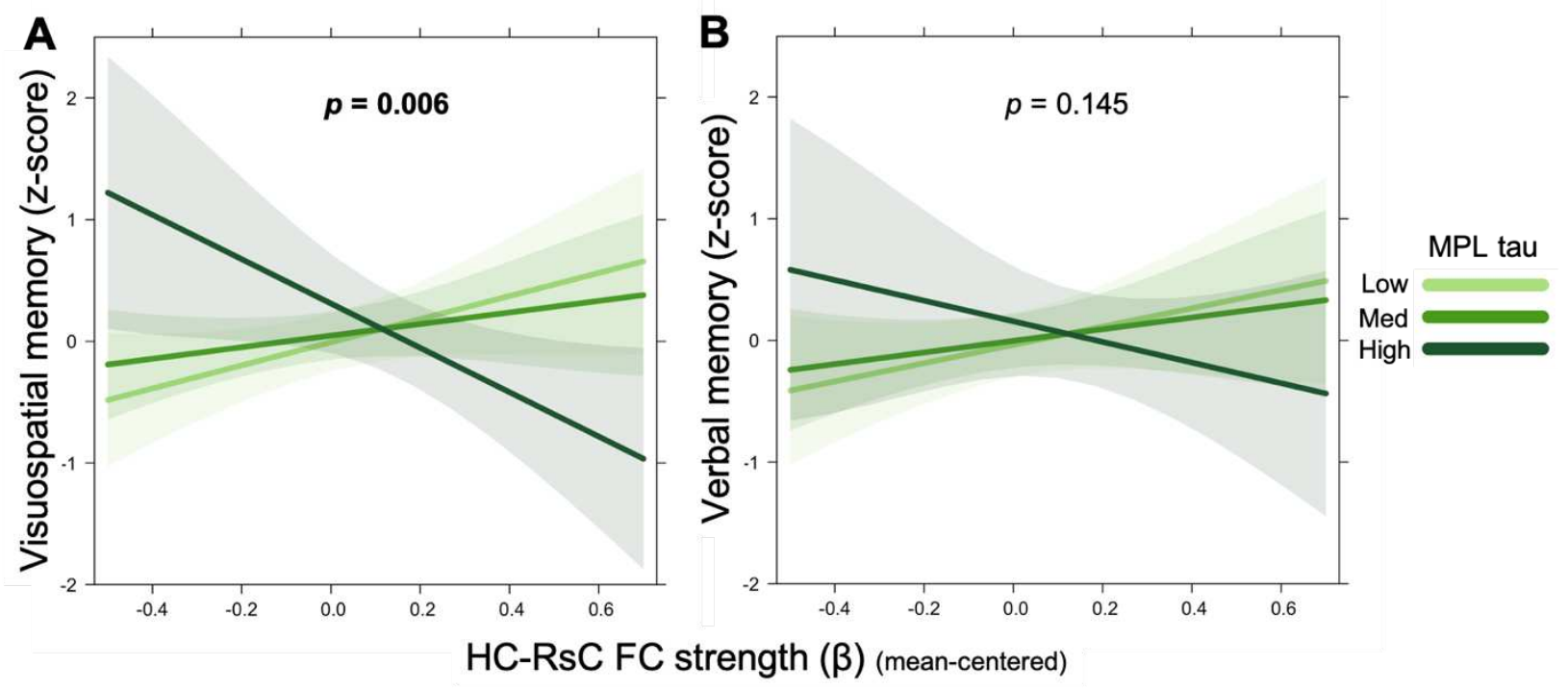
Figure 4. Episodic memory performance is associated with the interaction of hippocampal-retrosplenial connectivity and medial parietal tau. Visualization of interaction between hippocampal-retrosplenial (HC-RsC) functional connectivity (FC) strength (beta value) and medial parietal lobe (MPL) suprathreshold tau in relation to visuospatial and verbal memory, adjusting for age, sex, years of education, practice effects, and global A $\beta$. (A) Visuospatial memory (Visual Reproduction immediate and delay recall) is associated with the interaction of MPL tau and HC-RsC FC strength. (B) Verbal memory (CVLT immediate and long delay free recall) does not exhibit a significant interaction. Plots display the relationship predicted by linear regression at low $\left(10^{\text {th }}\right.$ percentile $)$, median, and high $\left(90^{\text {th }}\right.$ percentile) MPL suprathreshold tau.

Discussion

In this study of cognitively unimpaired older adults, we measured functional connectivity between the MTL and medial parietal lobe using resting state $\mathrm{MRI}$, and measured tau pathology

5 burden using tau PET imaging. Retrosplenial cortex exhibited strong functional connectivity with hippocampus, weaker yet significant connectivity with pmEC, and no connectivity with

7 alEC. We found that the strength of $\mathrm{HC}-\mathrm{RsC}$ connectivity was associated with the degree of tau accumulation in the medial parietal lobe, whereas neither alEC-RsC nor pmEC-RsC connectivity correlated with medial parietal lobe tau. Control analyses demonstrated that this result was

10 specific to $\mathrm{HC}-\mathrm{RsC}$ connectivity and to tau pathology in the downstream medial parietal lobe.

11 We further observed that the correspondence between tau in hippocampus and medial parietal

12 lobe was greater with stronger $\mathrm{HC}-\mathrm{RsC}$ connectivity. Finally, we demonstrated that greater 13 medial parietal lobe tau in combination with stronger HC-RsC connectivity was associated with

14 poorer episodic memory performance, particularly in the visuospatial domain. Together, these

15 findings provide strong evidence that AD-related tau pathology disrupts memory processing by

16 spreading first from entorhinal cortex to hippocampus before later accumulating in medial

17 parietal lobe. The combination of high MTL and medial parietal tau and high functional

18 connectivity between these regions in cognitively normal individuals may thus represent the

19 conditions under which the earliest stages of cognitive decline occur. 
2 Tau accumulates in medial parietal lobe via connectivity with hippocampus

3 These results add to a growing literature linking patterns of resting state functional

4 connectivity to the spread of AD-related tau pathology. In the aging brain, greater functional

5 connectivity between regions is related to greater tau accumulation and correspondence of tau

6 between these regions ${ }^{11,12}$. Further, models predicting the spread of tau using functional

7 connectivity closely resemble the observed pattern of tau deposition in the brain ${ }^{7,8}$, and

8 functional connectivity may even be a better predictor of tau propagation than traditional Braak

9 staging ${ }^{13}$. Our finding that connectivity between retrosplenial cortex and hippocampus, but not

10 alEC or pmEC, was associated with medial parietal tau suggests that tau originating in MTL

11 accumulates in hippocampus before later spreading directly to medial parietal lobe via

12 connectivity with retrosplenial cortex. In addition, the finding that pmEC-RsC connectivity was

13 weaker than $\mathrm{HC}-\mathrm{RsC}$ connectivity indicates that although pmEC is functionally connected with

14 several posterior medial areas ${ }^{11,33}$, it is not likely to be the primary structure involved in the

15 propagation of tau to the medial parietal lobe. Instead, tau likely spreads to alEC-connected

16 neocortex and hippocampus at a comparable rate, and later accumulates in posterior medial areas

17 with connections to the hippocampus. This is consistent with cross-sectional histopathological

18 data indicating that although the earliest cortical region to exhibit tau pathology is the

19 alEC/transentorhinal area ${ }^{14,34}$, tau is typically observed in hippocampus prior to limbic areas

20 such as the retrosplenial region ${ }^{15}$. These findings also corroborate recent work that found that

21 structural connectivity between hippocampus and posterior cingulate cortex (PCC) was

22 associated with tau pathology in $\mathrm{PCC}^{10}$. 
The specificity of this key finding in our study is striking. The lack of association

2 between $\mathrm{HC}-\mathrm{RsC}$ connectivity and tau in entorhinal cortex suggests this connectivity is

3 specifically related to tau in the downstream medial parietal region of this pathway. In addition

4 to the medial parietal lobe, the inferior temporal cortex is one of the first areas outside of the

5 MTL where tau pathology begins to accumulate, and tau burden in this region is often used as a

6 marker of AD disease progression ${ }^{29,30}$. That the strength of HC-RsC connectivity was only

7 associated at trend level with tau in this region suggests that this connectivity is specific to tau

8 pathology in the medial parietal lobe and not in other early-accumulating neocortical areas. In

9 addition, though hippocampus showed strong functional connectivity with the medial portion of

10 Brodmann area 10, a region of the default mode network in the superior frontal gyrus, there was

11 no association between the strength of this connectivity and medial parietal tau. Thus, it appears

12 that connectivity specifically between hippocampus and retrosplenial cortex is associated with

13 medial parietal tau, not simply connectivity between hippocampus and other highly-connected

14 cortical area. Finally, we failed to find an association between $\mathrm{HC}-\mathrm{RsC}$ and $\mathrm{A} \beta$ burden within

15 medial parietal lobe. This is not surprising given that $A \beta$ does not originate in the MTL and is

16 not thought to spread in the same transneuronal manner as tau, lending further support to the

17 notion that functional connectivity is uniquely useful in predicting tau accumulation. Taken

18 together, these results support a narrative of tau pathology in hippocampus spreading to medial

19 parietal lobe in cognitively unimpaired older adults, reflected by resting state functional

20 connectivity between these regions.

22 Functional connectivity may modulate tau propagation at different time scales 
In addition to the association between functional connectivity and tau pathology, our

2 finding of greater correspondence between hippocampal and medial parietal tau with greater HC-

3 RsC connectivity strength lends further support to the notion that neural connectivity may

4 influence tau to spread from early-accumulating regions to connected downstream areas. In

5 particular, individuals with greater connectivity strength in our sample were more likely to have

6 tau pathology burden in hippocampus align with medial parietal lobe tau than individuals with

7 weaker connectivity. It is notable that although replicating this analysis using entorhinal tau PET

8 demonstrated this same relationship at trend level, the strongest relationship was evident with

9 hippocampal tau. Because tau in entorhinal cortex and hippocampus tend to be highly correlated

10 with one another, it is not surprising that using entorhinal tau yielded a similar result, and further

11 suggests that contamination of hippocampal signal from non-specific choroid plexus FTP

12 binding is not likely to be driving this finding.

13 An alternative explanation for these findings is that tau accumulation influences the

14 degree of functional connectivity between these regions. Indeed, some studies have found a

15 negative association between tau pathology and functional connectivity across the cortex $29,35,36$,

16 though these tended to use global measures of tau or focused on A $\beta$-positive individuals.

17 However, our findings together with previous work help illustrate how tau and functional

18 connectivity may produce distinct effects at different time scales. Initially, greater connectivity

19 within an individual circuit may facilitate the spread of tau from regions of early accumulation to

20 downstream cortical areas, perhaps enhanced by the presence of $A \beta$. Over time, however, the

21 influence of tau throughout this pathway leads to neurodegeneration and disruption of neuronal

22 signaling, and tau pathology may in fact show an inverse correlation with functional

23 connectivity. These distinct short- and long-term effects may help explain regional differences in 
1 the tau-connectivity relationship such that pathways of early tau propagation are first to show

2 local degeneration, whereas later pathways, such as the MTL-medial parietal lobe, may

3 concurrently demonstrate increased connectivity leading to further tau spread. In the context of a

4 cascading network failure model of $\mathrm{AD}^{37}$, functional connectivity has proven to be a powerful

5 tool in examining the effect of tau accumulation on functional isolation of brain regions ${ }^{32}$ and in

6 predicting inter-individual variability in the pattern of tau spread and disease progression ${ }^{13}$.

8 Greater connectivity and tau together lead to worse episodic memory

9 In this study, functional connectivity and tau were also found to have consequences for

10 cognitive function. Episodic memory performance, particularly visuospatial memory, was related

11 to the combination of greater medial parietal lobe tau accumulation and greater HC-RsC

12 strength. Though neither connectivity strength nor medial parietal tau alone predicted memory

13 performance in our sample, it is notable that the interaction between them was associated with

14 poorer episodic memory in individuals without clinical cognitive impairment, even after

15 controlling for global $\mathrm{A} \beta$ burden. Existing work has yet to establish a clear relationship between

16 memory performance and connectivity changes in aging. Greater functional connectivity

17 between MTL and posterior medial areas is related to poorer memory performance both cross-

18 sectionally and over time ${ }^{36}$. By contrast, a positive association has been reported between MTL-

19 medial parietal connectivity and memory performance ${ }^{38,39}$, though these studies did not measure

20 tau pathology with PET imaging. Abnormal diffusivity of the hippocampal cingulum bundle has

21 also been shown to be related to greater decline in memory performance in older individuals with

22 high PCC tau and high A $\beta^{10}$. It may be that propagation of tau, not tau burden per se, is

23 associated with the earliest deficits in cognitive function, representing a state of mild circuit 
1 disruption that has not yet reached the stage of widespread neurodegeneration and cascading

2 network failure. This view comports with a model of AD stemming from peptide-dependent

3 circuit dysfunction, as tau propagation leads to changes in circuit excitability associated with the

4 earliest-detectable cognitive changes ${ }^{40}$. Indeed, the correlation between tau spread to the medial

5 parietal lobe and worse episodic memory performance may be an indicator of consequences for

6 the PM memory system, which while not affected by tau as early as the AT system may begin to

7 be disrupted in $\mathrm{MCI}$ and $\mathrm{AD}^{18}$.

8 It is also striking that our cognitive findings are driven by an association with visuospatial

9 memory in particular. Medial parietal areas have long been implicated in processing of spatial

10 information ${ }^{41-43}$, and the representation of visuospatial context is thought to be a key function of

11 the PM memory system ${ }^{24}$. Spatial information processing may also be one of the earliest-

12 affected domains in cognitive aging and Alzheimer's disease ${ }^{44}$, but the relationship between

13 functional connectivity and spatial memory performance has not been extensively studied in

14 humans. In rodents, RsC-PCC resting state functional connectivity has been shown to be

15 associated with impaired spatial memory performance ${ }^{45}$. Inferring a causal link between

16 propagation of tau to the medial parietal lobe and domain-specific cognitive decline is beyond

17 the scope of this study, but these processes may underlie the beginnings of cognitive decline in

18 healthy older adults.

\section{Limitations}

21 It should be noted that the cross-sectional nature of these data means that we can only

22 infer the spread of tau rather than directly observing it. Longitudinal studies of tau accumulation

23 are needed to fully validate the notion of tau propagating to different regions over time via 
1 connectivity. In addition, though we adjusted for A $\beta$ PET signal throughout this study, we did

2 not find that the relationships described here were stronger in individuals with greater global $A \beta$

3 burden. Though A $\beta$ was associated with medial parietal lobe tau independent of HC-RsC

4 connectivity, there was no significant interaction with $\mathrm{A} \beta$ in any of our analyses. This was a

5 somewhat surprising finding given a number of studies that have found a stronger association

6 between tau and connectivity for those with greater $A \beta$ pathology ${ }^{9-11}$. It is possible that our

7 sample did not provide us with enough statistical power to observe this interaction, though it was

8 enriched to include nearly half $\mathrm{A} \beta$-positive individuals. Another explanation is the processes

9 examined here represent features of normal aging independent of AD-specific pathologies.

10 Further study is needed to assess the role of $A \beta$ in the association between medial parietal lobe

11 tau and functional connectivity in cognitively normal older adults.

\section{Conclusions}

14 The findings described here support the view that tau pathology spreads from its origin in

15 the entorhinal cortex to the hippocampus in cognitively normal older individuals, before later

16 depositing in medial parietal lobe via direct connectivity between hippocampus and retrosplenial

17 cortex. Though the accumulation of tau pathology in MTL and even some areas of the AT

18 network has been observed in older adults without cognitive impairment, tau spread into the PM

19 network and subsequent domain-specific memory decline may reflect a significant transition

20 between normal aging and the processes involved in Alzheimer's disease. Future work with

21 longitudinal data can help establish tau propagation into the medial parietal lobe as a crucial

22 marker of the beginnings of Alzheimer's disease. 


\section{$1 \quad$ Materials and Methods}

2 Study Design

3 The main objective of this study was to determine what medial temporal lobe structure is

4 primarily involved in the propagation of tau into the medial parietal lobe in cognitively normal

5 older adults. We hypothesized that hippocampus would show the strongest functional

6 connectivity with medial parietal lobe, and that the strength of this connectivity would be

7 associated with the degree of medial parietal tau. After initial analysis, we further hypothesized

8 that the strength of this connectivity would modulate the correlation between medial temporal

9 and medial parietal tau, as well as between tau and episodic memory performance. To test these

10 hypotheses, we included data from 97 cognitively normal older adults from the Berkeley Aging

11 Cohort Study. All participants underwent 3T structural and resting state functional MRI, 3T

12 structural MRI, and a standard neuropsychological assessment. These participants also received

13 tau PET imaging using ${ }^{18} \mathrm{~F}$-Flortaucipir (FTP) and A $\beta$ PET imaging using ${ }^{11} \mathrm{C}$-Pittsburgh

14 Compound $\mathrm{B}(\mathrm{PiB})$. There were 3 individuals who did not have PiB PET data available for

15 analysis, and so were excluded from all analyses that adjusted for global A $\beta$ signal. We included

16 only participants whose resting state fMRI data was collected within 146 days of their

17 corresponding tau PET scan $(\mathrm{M}=42.5, \mathrm{SD}=37.9)$.

18 Additional inclusion criteria for this study were $60+$ years of age, cognitively normal

19 status (Mini Mental State Examination score $\geq 25$ and normal neuropsychological examination,

20 defined as within 1.5 SDs of age, education, and sex adjusted norms), no serious neurological,

21 psychiatric, or medical illness, no major contraindications found on MRI or PET, and

22 independent community living status. This study was approved by the Institutional Review 
1 Boards of the University of California, Berkeley, and the Lawrence Berkeley National

2 Laboratory (LBNL). All participants provided written informed consent.

4 3T MRI acquisition

5 Structural and functional MRI data were acquired on a 3T TIM/Trio scanner (Siemens

6 Medical System, software version B17A) using a 32-channel head coil. A T1-weighted whole

7 brain magnetization prepared rapid gradient echo (MPRAGE) image was acquired for each

8 subject (voxel size $=1 \mathrm{~mm}$ isotropic, $\mathrm{TR}=2300 \mathrm{~ms}, \mathrm{TE}=2.98 \mathrm{~ms}$, matrix $=256 \times 240 \times 160$, FOV

$9=256 \times 240 \times 160 \mathrm{~mm}^{3}$, sagittal plane, 160 slices, $5 \mathrm{~min}$ acquisition time). Resting state functional

10 MRI was then acquired using T2*-weighted echo planar imaging (EPI, voxel size $=2.6 \mathrm{~mm}$

11 isotropic, $\mathrm{TR}=1.067 \mathrm{~ms}, \mathrm{TE}=31.2 \mathrm{~ms}, \mathrm{FA}=45$, matrix $80 \times 80, \mathrm{FOV}=210 \mathrm{~mm}$, sagittal plane,

12300 volumes, anterior to posterior phase encoding, ascending acquisition, 5 min acquisition

13 time). During resting state acquisition, participants were told to remain awake with eyes open

14 and focused on a white asterisk displayed on a black background.

16 Structural MRI preprocessing

17 Structural T1-weighted images were processing using Statistical Parametric Mapping

18 (SPM12). Images were first segmented into gray matter, white matter, and CSF components in

19 native space. DARTEL-imported tissue segments for all individuals in the sample were used to

20 create a study-specific template, which was then used to warp native space T1 images and tissue

21 segments to MNI space at $2 \mathrm{~mm}$ isotropic resolution. Finally, native space $\mathrm{T} 1$ images were

22 segmented with Freesurfer v.5.3.0 using the Desikan-Killany atlas parcellation. 
11 carried out using the CONN functional connectivity toolbox (version 17e) ${ }^{46}$ implemented in

12 MATLAB version 2019b (The Mathworks Inc., Natick, MA). ART motion detection was first

13 performed to identify volumes of high motion, using a movement threshold of $>0.5 \mathrm{~mm} / \mathrm{TR}$ and a

14 global intensity z-score of 3. Outlier volumes were flagged and included as spike regressors

15 during denoising. No individuals were excluded from these analyses due to excess motion, as all

16 participants had $<20 \%$ of outlier volumes $(\mathrm{M}=5.0 \%, \mathrm{SD}=3.4 \%)$. Denoising was then

17 performed with translation and rotation realignment parameters and their first-order derivatives,

18 as well as anatomical CompCor (first five components of time series signal from white matter

19 and CSF). A band pass filter of $0.008-0.1 \mathrm{~Hz}$ and linear detrending were then applied to the

20 residual time-series.

22 Regions of interest for functional connectivity analyses 
A number of regions of interest (ROI) were defined for these analyses to examine the

2 association of functional connectivity between these areas and tau burden. Many of these were

3 obtained from the FreeSurfer segmentation of each participant's native space 3T structural image

4 47. The regions defined in this way included the hippocampus (HC), retrosplenial cortex (RsC),

5 posterior cingulate cortex (PCC), precuneus (PrC), whole entorhinal cortex (EC), and inferior

6 temporal cortex (IT). In the case of RsC, we used the FreeSurfer region analog labeled as

7 “isthmus cingulate." The composite medial parietal lobe ROI used throughout this study

8 consisted of the $\mathrm{RsC}, \mathrm{PCC}$, and $\mathrm{PrC}$ regions. To test connectivity between $\mathrm{HC}$ and a region

9 outside of the medial parietal lobe, we also identified a region in the superior frontal gyrus (SFG)

10 analogous to the medial portion of Brodman Area 10, labeled as A10m in the Brainnetome atlas

11 parcellation ${ }^{28}$.

12 Anterolateral entorhinal cortex (alEC) and posteromedial entorhinal cortex (pmEC) were

13 defined in a previous study with high-resolution 7T MRI ${ }^{27}$. In brief, anatomical borders of the

14 entire entorhinal cortex were manually defined on a high-resolution T1-group template.

15 Multivariate classification in a group of young adults was used to then identify clusters of voxels

16 within this mask that showed preferential functional connectivity with perirhinal cortex,

17 comprising the alEC ROI, or with the parahippocampal gyrus, comprising the pmEC ROI. These

18 alEC and pmEC ROIs were then warped to a $2 \mathrm{~mm}$ isotropic MNI template and made publicly

19 available. In this study, we used these bilateral MNI space ROIs in our functional connectivity

20 analyses. Because these regions are in close spatial proximity to one another, we extracted time

21 series from the unsmoothed, denoised MNI space resting state data to avoid smoothing signal

22 from each seed into each other. 
To address signal dropout in these and every ROI in these analyses, we derived an

2 explicit mask to remove regions of low signal across the whole brain. This mask was defined by

3 calculating the mean functional MNI space image across all individuals, restricted to a group

4 level grey matter mask. We then excluded voxels with less than $40 \%$ of the mean signal intensity

5 of the image. Using this mean signal intensity threshold mask, a mean of $15.8 \%$ of voxels $(\mathrm{SD}=$

$6 \quad 11.9 \%$ ) were removed across all ROIs with the highest proportion of voxels being removed from

7 the A10m region (34.8\%).

$9 \quad$ Functional connectivity analysis

10 Seed-to-seed functional connectivity analysis was carried out with the CONN toolbox ${ }^{46}$

11 using the MNI space resting state fMRI data. We used semipartial correlations for all first-level

12 analyses to compute the time series correlation between each seed, controlling for the variance of

13 all other seed regions entered into the same model. We first constructed a model of functional

14 connectivity between alEC, pmEC, HC, and RsC using bilateral ROIs. Semipartial correlations

15 and unsmoothed data were used to minimize spillover of signal between adjacent MTL regions.

16 Statistical significance of functional connectivity was determined using one-sample t-tests of $\beta$ -

17 weights from region-to-region semipartial correlations to see if connectivity was significantly

18 different from 0 . All analyses were performed using an explicit mask to remove areas of high

19 signal dropout across the whole brain, as described above.

$21 \quad$ PET acquisition and processing

22 PET was acquired for all participants at LBNL. Tau accumulation was assessed with ${ }^{18} \mathrm{~F}-$

23 Flortaucipir (FTP) synthesized at the Biomedical Isotope Facility at LBNL as previously 
1 described ${ }^{3}$. Data were collected on a Biograph TruePoint 6 scanner (Siemens, Inc) 75-115 min

2 post-injection in listmode. Data were then binned into 4 x 5 min frames from $80-100$ min post-

3 injection. CT scans were performed before the start of each emission acquisition. A $\beta$ burden was

4 assessed using ${ }^{11} \mathrm{C}$-Pittsburgh Compound $\mathrm{B}(\mathrm{PiB})$, also synthesized at the Biomedical Isotope

5 Facility at LBNL ${ }^{48}$. Data were collected on the Biograph scanner across 35 dynamic frames for

690 min post-injection and subsequently binned into 35 frames $(4 \times 15,8 \times 30,9 \times 60,2 \times 180,10$

7 x 300, and 2 x 600s), and a CT scan was performed. All PET images were reconstructed using an

8 ordered subset expectation maximization algorithm, with attenuation correction, scatter

9 correction, and smoothing with a $4 \mathrm{~mm}$ Gaussian kernel.

10 Processing of FTP images was carried out in SPM12. Images were realigned, averaged,

11 and coregistered to 3T structural MRIs. Standardized uptake value ratio (SUVR) images were

12 calculated by averaging mean tracer uptake over the 80-100 min data and normalized with an

13 inferior cerebellar gray reference region ${ }^{31}$. The mean SUVR of each ROI (structural MRI

14 FreeSurfer segmentation) was extracted from the native space images. This ROI data was partial

15 volume corrected using a modified Geometric Transfer Matrix approach ${ }^{49}$ as previously

16 described ${ }^{31}$. SUVR images were then warped to $2 \mathrm{~mm}$ MNI space for voxelwise analyses using

17 the study-specific DARTEL template produced from structural data (see above). No additional

18 spatial smoothing was applied.

19 Using SPM12, PiB images were realigned. An average of frames within the first $20 \mathrm{~min}$

20 was used to calculate the transformation matrix to coregister the PiB images to the participants'

$213 \mathrm{~T}$ structural MRI; this transformation matrix was then applied to all PiB frames. Distribution

22 volume ratio (DVR) images were calculated with Logan graphical analysis over 35-90 min data

23 and normalized to a whole cerebellar gray reference region ${ }^{50,51}$. Global PiB was calculated 
1 across cortical FreeSurfer ROIs as previously described ${ }^{52}$, and a threshold of DVR $>1.065$ was

2 used categorize participants as $\mathrm{A} \beta$-positive or $\mathrm{A} \beta$-negative. In addition, mean DVR within each

3 FreeSurfer ROI was extracted from coregistered, MNI space PiB images.

\section{Suprathreshold tau quantification}

To quantify tau deposition, we used the proportion of voxels above an a priori threshold

7 of SUVR $>1.4$ for FTP PET signal. This suprathreshold tau measure has previously been shown

8 to be a reliable marker of AD-related tau pathology ${ }^{23}$, and has been used in previous studies

9 investigating functional connectivity and tau ${ }^{11}$. One distinct advantage of using suprathreshold

10 FTP over mean SUVR is that it is not confounded by different number of voxels within ROIs.

11 For each individual, we computed the number of suprathreshold FTP voxels within each ROI

12 and divided by the total number of voxels in the region.

13

14 Statistical analysis

15 All statistical analysis was carried out in $\mathrm{R}$ version 3.6.3, with a two-sided significance

16 level of $\alpha=0.05$ throughout. We assessed the relationship between tau, resting state functional

17 connectivity, and cognitive function in our sample using linear regression models carried out

18 with the $\operatorname{lm}()$ function in the stats $\}$ package. All analyses were adjusted for age at time of tau

19 scan, sex, and mean global A $\beta$ burden. Analyses involving cognitive test performance were

20 additionally adjusted for years of education as well as practice effects quantified as the square

21 root of the number of prior testing occasions ${ }^{53}$.

23 Cognitive measures 
To assess episodic memory in our sample of cognitively normal older adults, we used

2 neuropsychological assessment data from closest in time to each individual's tau scan. There was

3 a mean of 84.2 days $(\mathrm{SD}=56.9)$ between each individual's cognitive assessment and tau PET

4 scan. We computed an episodic memory composite measure by averaging the z-transformed

5 individual test scores using mean and SD from the sample ${ }^{32,54}$ for four different tasks. These

6 tasks were the California Verbal Learning Test (CVLT) immediate free recall, CVLT long-delay

7 free recall, Visual Reproduction I (immediate recall), and Visual Reproduction II (delay recall).

8 We analyzed distinct verbal and visuospatial episodic memory performance by considering

9 performance in CVLT and Visual Reproduction tasks separately.

\section{Supplementary Materials}

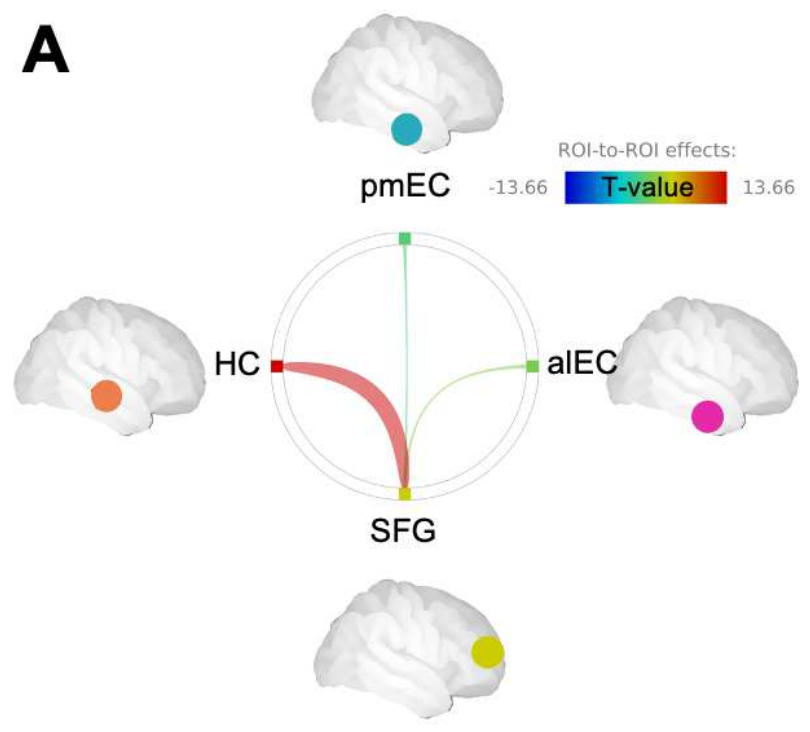

12

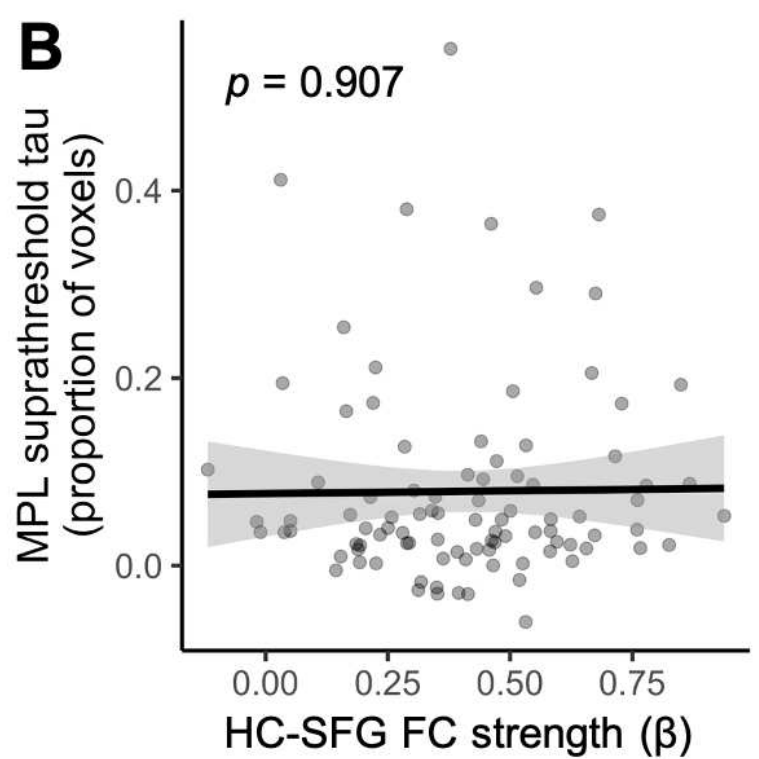


Supplementary Figure 1. Hippocampal-retrosplenial resting state functional connectivity is associated with medial parietal lobe tau accumulation. (A) Superior frontal gyrus (SFG; medial portion of Brodmann Area 10) exhibits resting state functional connectivity with hippocampus $(\beta=0.40, p<0.001)$, but not with posteromedial entorhinal cortex $(\beta=-0.02, p=0.379)$ or anterolateral entorhinal cortex $(\beta=0.02, p=0.439)$. Line color and thickness correspond to the T-statistic of semipartial correlations of resting state activity between regions. (B) Adjusting for age, sex, and global A $\beta$, HC-SFG functional connectivity strength is not associated with proportion of voxels above threshold (SUVR > 1.4) within medial parietal lobe. Connectivity strength measured by extracting $\beta$-values of semipartial correlations between hippocampus and SFG.

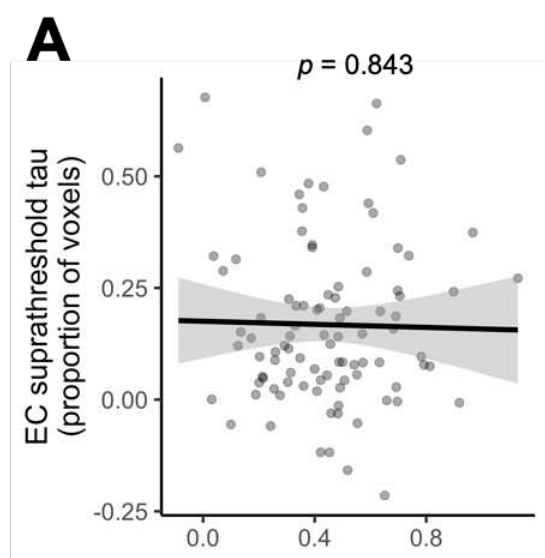

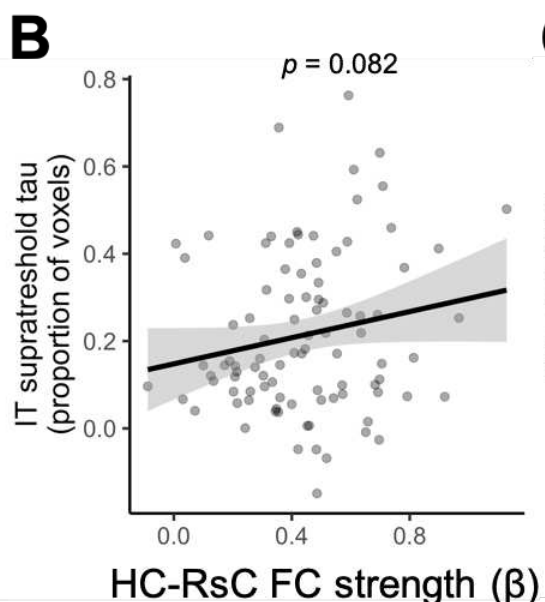

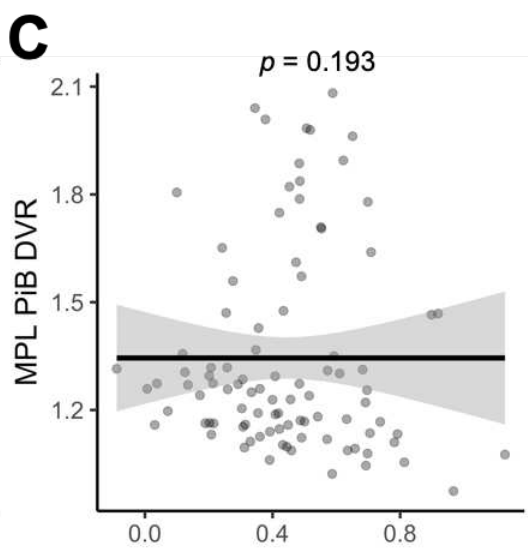

HC-RsC FC strength $(\beta)$

Supplementary Figure 2. Hippocampal-retrosplenial resting state functional connectivity is specifically associated with medial parietal lobe tau accumulation. (A) HC-RsC is not associated with entorhinal cortex (EC) suprathreshold tau. (B) HC-RsC shows a trend-level association with inferior temporal (IT) suprathreshold tau. (C) HC-RsC is not associated with MPL A $\beta$ measured by PiB PET DVR. Plots show residualized values from linear regression models adjusting for age, sex, and global A $\beta$. Connectivity strength measured by extracting $\beta$ values of semipartial correlations between $\mathrm{HC}$ and $\mathrm{RsC}$. Suprathreshold tau defined as proportion of voxels above threshold (SUVR > 1.4) within each region of interest.

Supplementary Table 1. Model results. Examined variables from linear regression model examining the relationship between medial parietal lobe (MPL) suprathreshold tau and age, sex, global beta amyloid (A $\beta$ ), choroid plexus signal (partial volume corrected FTP SUVR), entorhinal mean tau (partial volume corrected FTP SUVR), hippocampal-retrosplenial (HC$\mathrm{RsC}$ ) functional connectivity (FC) strength, and entorhinal mean tau x HC-RsC FC strength interaction. Bolded term indicates interaction effect visualized in Supplementary Figure 3.

Independent variables: $\quad$ Dependent variable: MPL suprathreshold tau 


$\begin{array}{ll}\text { age } & \beta=-0.002 \\ \text { sex } & p=0.373 \\ \text { global A } \beta & \beta=0.011 \\ & \mathrm{p}=0.614 \\ \text { choroid plexus signal } & \beta=0.033 \\ & \mathrm{p}=0.523 \\ \text { entorhinal mean tau } & \beta=0.098 \\ & \mathrm{p}=0.026 \\ \text { HC-RsC FC strength } & \beta=0.116 \\ & \mathrm{p}=0.027 \\ \text { entorhinal mean tau } \mathbf{x} \text { HC-RsC } & \beta=0.130 \\ \text { FC strength } & \mathrm{p}=0.005 \\ \text { Constant } & \mathbf{\beta}=\mathbf{0 . 2 6 5} \\ & \mathbf{p}=\mathbf{0 . 0 6 9} \\ & \beta=-0.106 \\ & \mathrm{p}=0.209\end{array}$

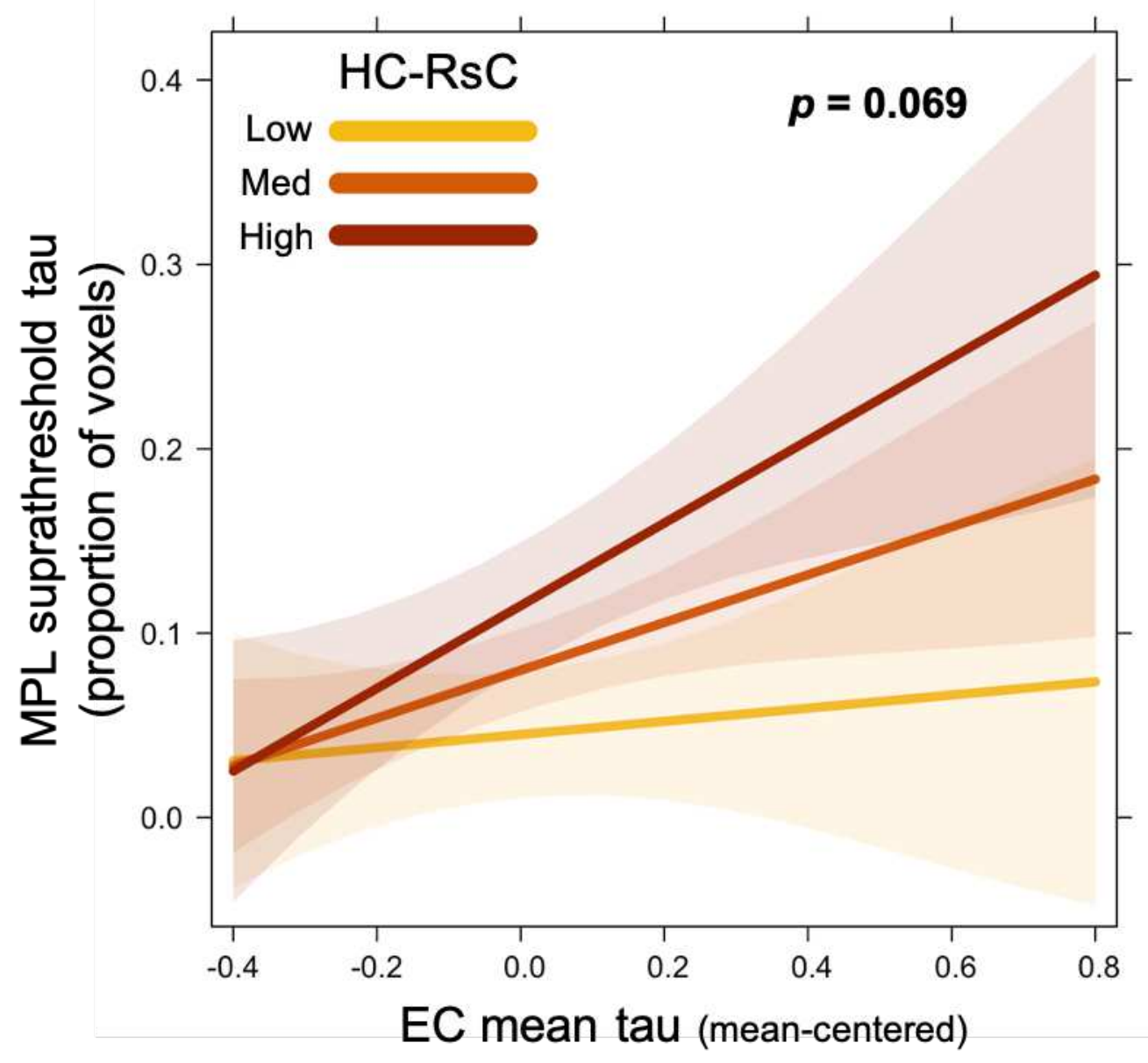




\section{Supplementary Figure 3. Entorhinal tau pathology exhibits greatest association with medial parietal lobe tau when hippocampal-retrosplenial connectivity is greatest. Visualization of interaction between entorhinal cortex (EC) mean tau (partial volume corrected FTP SUVR) and hippocampal-retrosplenial functional connectivity strength (HC-RsC) from linear regression model (see Supplementary Table 1). Medial parietal lobe (MPL) suprathreshold tau is associated with an entorhinal tau x HC-RsC interaction at trend level. Plot displays the relationship predicted by linear regression at low $\left(10^{\text {th }}\right.$ percentile $)$, median, and high $\left(90^{\text {th }}\right.$ percentile) HC-RsC.}

3 References:

4 1. Braak, H. \& Braak, E. Neuropathological stageing of Alzheimer-related changes. Acta $5 \quad$ Neuropathol. 239-259 (1991) doi:10.1007/BF00308809.

6 2. Johnson, K. A. et al. Tau positron emission tomographic imaging in aging and early Alzheimer disease. Ann. Neurol. 79, 110-119 (2016).

8 3. Schöll, M. et al. PET Imaging of Tau Deposition in the Aging Human Brain. Neuron 89, 9 971-982(2016).

10 4. Pooler, A. M., Phillips, E. C., Lau, D. H. W., Noble, W. \& Hanger, D. P. Physiological 11 release of endogenous tau is stimulated by neuronal activity. EMBO Rep. 14, 389-394 (2013).

13 5. Ahmed, Z. et al. A novel in vivo model of tau propagation with rapid and progressive neurofibrillary tangle pathology: The pattern of spread is determined by connectivity, not 15 proximity. Acta Neuropathol. 127, 667-683 (2014).

166 Wu, J. W. et al. Neuronal activity enhances tau propagation and tau pathology in vivo.

$17 \quad$ Nat. Neurosci. 19, 1085-1092 (2016).

187 Cope, T. E. et al. Tau burden and the functional connectome in Alzheimer's disease and progressive supranuclear palsy. (2018) doi:10.1093/brain/awx347. 
1 8. Vogel, J. W. et al. Spread of pathological tau proteins through communicating neurons in

2 human Alzheimer's disease. Nat. Commun. 11, 2612 (2020).

39 . Franzmeier, N. et al. Functional brain architecture is associated with the rate of tau

4 accumulation in Alzheimer's disease Nicolai. Nat. Commun. 11, 1-17 (2020).

5 10. Jacobs, H. I. L. et al. Structural tract alterations predict downstream tau accumulation in

6 amyloid-positive older individuals. Nat. Neurosci. 21, 424-431 (2018).

7 11. Adams, J. N., Maass, A., Harrison, T. M., Baker, S. L. \& Jagust, W. J. Cortical tau

8 deposition follows patterns of entorhinal functional connectivity in aging. Elife 8, 1-22

9 (2019).

10 12. Franzmeier, N. et al. Functional connectivity associated with tau levels in ageing,

11 Alzheimer's, and small vessel disease. Brain 1093-1107 (2019)

12 doi:10.1093/brain/awz026.

13 13. Franzmeier, N. et al. Patient-centered connectivity-based prediction of tau pathology

14 spread in Alzheimer's disease. Sci. Adv. 6, (2020).

15 14. Braak, H. \& Braak, E. On areas of transition between entorhinal allocortex and temporal

16 isocortex in the human brain. Normal morphology and lamina-specific pathology in

17 Alzheimer's disease. Acta Neuropathol. 68, 325-332 (1985).

18 15. Braak, H. \& Braak, E. Staging of alzheimer's disease-related neurofibrillary changes.

$19 \quad$ Neurobiol. Aging 16, 271-278 (1995).

20 16. Crary, J. F. et al. Primary age-related tauopathy (PART): a common pathology associated

21 with human aging. Acta Neuropathol. 128, 755-766 (2014).

22 17. He, Z. et al. Amyloid- $\beta$ plaques enhance Alzheimer's brain tau-seeded pathologies by

23 facilitating neuritic plaque tau aggregation. Nat. Med. 24, 29-38 (2018). 
1 18. Maass, A. et al. Alzheimer's pathology targets distinct memory networks in the ageing $2 \quad$ brain. Brain 142, 2492-2509 (2019).

3 19. Harrison, T. M. et al. Longitudinal tau accumulation and atrophy in aging and Alzheimer 4 disease. Ann. Neurol. 85, 229-240 (2019).

5 20. Van Hoesen, G. W., Pandya, D. N. \& Butters, N. Some connections of the entorhinal (area 628 ) and perirhinal (area 35) cortices of the rhesus monkey. II. Frontal lobe afferents. Brain $7 \quad$ Res. 95, 25-38 (1975).

8 21. Witter, M. P., Groenewegen, H. J., Lopes da Silva, F. H. \& Lohman, A. H. M. Functional 9 organization of the extrinsic and intrinsic circuitry of the parahippocampal region. Prog. $10 \quad$ Neurobiol. 33, 161-253 (1989).

11 22. Minoshima, S. et al. Metabolic reduction in the posterior cingulate cortex in very early 12 Alzheimer's disease. Ann. Neurol. 42, 85-94 (1997).

13 23. Maass, A. et al. Comparison of multiple tau-PET measures as biomarkers in aging and 14 Alzheimer's disease. Neuroimage 157, 448-463 (2017).

15 24. Ranganath, C. \& Ritchey, M. Two cortical systems for memory-guided behaviour. Nat. 16 Rev. Neurosci. 13, 713-726 (2012).

17 25. Ritchey, M., Libby, L. A. \& Ranganath, C. Cortico-hippocampal systems involved in 18 memory and cognition: The PMAT framework. Progress in Brain Research vol. 219 19 (Elsevier B.V., 2015).

20 26. Chrastil, E. R. Heterogeneity in human retrosplenial cortex: A review of function and 21 connectivity. Behav. Neurosci. 132, 317-338 (2018).

22 27. Maass, A., Berron, D., Libby, L. A., Ranganath, C. \& Düzel, E. Functional subregions of 23 the human entorhinal cortex. Elife 4, 1-20 (2015). 
1 28. Fan, L. et al. The Human Brainnetome Atlas: A New Brain Atlas Based on Connectional

$2 \quad$ Architecture. Cereb. Cortex 26, 3508-3526 (2016).

3 29. Schultz, A. P. et al. Phases of Hyperconnectivity and Hypoconnectivity in the Default

$4 \quad$ Mode and Salience Networks Track with Amyloid and Tau in Clinically Normal

$5 \quad$ Individuals. J. Neurosci. 37, 4323-4331 (2017).

630 . Huijbers, W. et al. Tau accumulation in clinically normal older adults is associated with

7 hippocampal hyperactivity. J. Neurosci. (2018) doi:10.1523/JNEUROSCI.1397-18.2018.

8 31. Baker, S. L., Maass, A. \& Jagust, W. J. Considerations and code for partial volume

9 correcting $\left[{ }^{18} \mathrm{~F}\right]-\mathrm{AV}-1451$ tau PET data. Data Br. 15, 648-657 (2017).

10 32. Harrison, T. M. et al. Tau deposition is associated with functional isolation of the

11 hippocampus in aging. Nat. Commun. 10, (2019).

12 33. Schröder, T. N., Haak, K. V., Jimenez, N. I. Z., Beckmann, C. F. \& Doeller, C. F.

13 Functional topography of the human entorhinal cortex. Elife 4, 1-17 (2015).

14 34. Kaufman, S. K., Del Tredici, K., Thomas, T. L., Braak, H. \& Diamond, M. I. Tau seeding

15 activity begins in the transentorhinal/entorhinal regions and anticipates phospho-tau

16 pathology in Alzheimer's disease and PART. Acta Neuropathol. 136, 57-67 (2018).

17 35. Sepulcre, J. et al. Tau and amyloid- $\beta$ proteins distinctively associate to functional network

18 changes in the aging brain. Alzheimer's Dement. 1-9 (2017)

19 doi:10.1016/j.jalz.2017.02.011.

20 36. Berron, D., van Westen, D., Ossenkoppele, R., Strandberg, O. \& Hansson, O. Medial

21 temporal lobe connectivity and its associations with cognition in early Alzheimer's

22 disease. Brain (2020) doi:10.1093/brain/awaa068.

23 37. Jones, D. T. et al. Tau, amyloid, and cascading network failure across the Alzheimer's 
2 38. Wang, L. et al. Intrinsic connectivity between the hippocampus and posteromedial cortex

disease spectrum. Cortex 97, 143-159 (2017). predicts memory performance in cognitively intact older individuals. Neuroimage $\mathbf{5 1}$, 910-917 (2010).

39. Kaboodvand, N., Bäckman, L., Nyberg, L. \& Salami, A. The retrosplenial cortex: A memory gateway between the cortical default mode network and the medial temporal lobe. Hum. Brain Mapp. 39, 2020-2034 (2018).

40. Harris, S. S., Wolf, F., De Strooper, B. \& Busche, M. A. Tipping the Scales: PeptideDependent Dysregulation of Neural Circuit Dynamics in Alzheimer's Disease. Neuron 119 (2020) doi:10.1016/j.neuron.2020.06.005.

41. Flicker, C., Bartus, R. T., Crook, T. H. \& Ferris, S. H. Effects of aging and dementia upon recent visuospatial memory. Neurobiol. Aging 5, 275-283 (1984).

42. Bushara, K. O. et al. Modality-specific frontal and parietal areas for auditory and visual spatial localization in humans. Nat. Neurosci. 2, 759-766 (1999).

43. Iachini, T., Iavarone, A., Senese, V., Ruotolo, F. \& Ruggiero, G. Visuospatial Memory in Healthy Elderly, AD and MCI: A Review. Curr. Aging Sci. 2, 43-59 (2012).

44. Lithfous, S., Dufour, A. \& Després, O. Spatial navigation in normal aging and the prodromal stage of Alzheimer's disease: Insights from imaging and behavioral studies. Ageing Res. Rev. 12, 201-213 (2013).

45. Ash, J. A. et al. Functional connectivity with the retrosplenial cortex predicts cognitive aging in rats. Proc. Natl. Acad. Sci. U. S. A. 113, 12286-12291 (2016).

46. Whitfield-Gabrieli, S. \& Nieto-Castanon, A. Conn: A Functional Connectivity Toolbox for Correlated and Anticorrelated Brain Networks. Brain Connect. 2, 125-141 (2012). 
1 47. Desikan, R. S. et al. An automated labeling system for subdividing the human cerebral cortex on MRI scans into gyral based regions of interest. Neuroimage 31, 968-980 (2006).

3 48. Mathis, C. A. et al. Synthesis and evaluation of 11C-labeled 6-substituted 2-

4 arylbenzothiazoles as amyloid imaging agents. J. Med. Chem. 1-15 (2009).

5 49. Rousset, O. G., Ma, Y. \& Evans, A. C. Correction for partial volume effects in PET:

6 Principle and validation. J. Nucl. Med. 39, 904-911 (1998).

7 50. Logan, J. Graphical analysis of PET data applied to reversible and irreversible tracers. $8 \quad$ Nucl. Med. Biol. 27, 661-670 (2000).

9 51. Price, J. C. et al. Kinetic modeling of amyloid binding in humans using PET imaging and 10 Pittsburgh Compound-B. J. Cereb. Blood Flow Metab. 25, 1528-1547 (2005).

11 52. Mormino, E. C. et al. Not quite PIB-positive, not quite PIB-negative: Slight PIB 12 elevations in elderly normal control subjects are biologically relevant. Neuroimage 59, $13 \quad 1152-1160(2012)$.

14 53. Vonk, J. M. J. et al. Semantic loss marks early Alzheimer's disease-related neurodegeneration in older adults without dementia. Alzheimer's Dement. Diagnosis, Assess. Dis. Monit. 12, 1-14 (2020).

17 54. Maass, A. et al. Entorhinal tau pathology, episodic memory decline, and neurodegeneration in aging. J. Neurosci. 38, 530-543 (2018).

Acknowledgments: We would like to thank R. La Joie for consultation on selecting regions of

21 interest and visualization of results. This work was supported by NIH grants T32-NS095939

22 (J.Z.), F31-AG062090 (J.N.A), R01-AG062542 (W.J.J.) and R01-AG034570 (W.J.J.), and by 23 the Rainwater Charitable Foundation (W.J.J.). 
1 Author Contributions: J.Z. carried out all analyses associated with the study, was responsible

2 for final experimental design decisions, and wrote the manuscript. J.N.A oversaw connectivity

3 analyses, provided some example scripts for the processing and analysis of data, and gave

4 meaningful feedback and consultation throughout. T.M.H. provided consultation for resting state

5 analyses and provided feedback on design and manuscript. S.L.B. provided PET imaging and

6 general methodology expertise, was involved in collection and preprocessing of PET data, and

7 gave manuscript feedback. W.J.J. conceptualized project, gave guidance and feedback

8 throughout, and provided data and funding for the project.

9 Competing Interests: W.J.J. consults for Genentech, Biogen, and Bioclinica.

10 Materials and Correspondence: All communications and request for materials should be

11 directed to the corresponding author (J.Z., jacob_ziontz@berkeley.edu). Suprathreshold medial

12 parietal tau image is available for viewing at the following NeuroVault repository:

13 https://neurovault.org/collections/9317. The code supporting the current study has not been

14 deposited in a public repository because it contains participants' identifying information but is

15 available on request. 


\section{Figures}

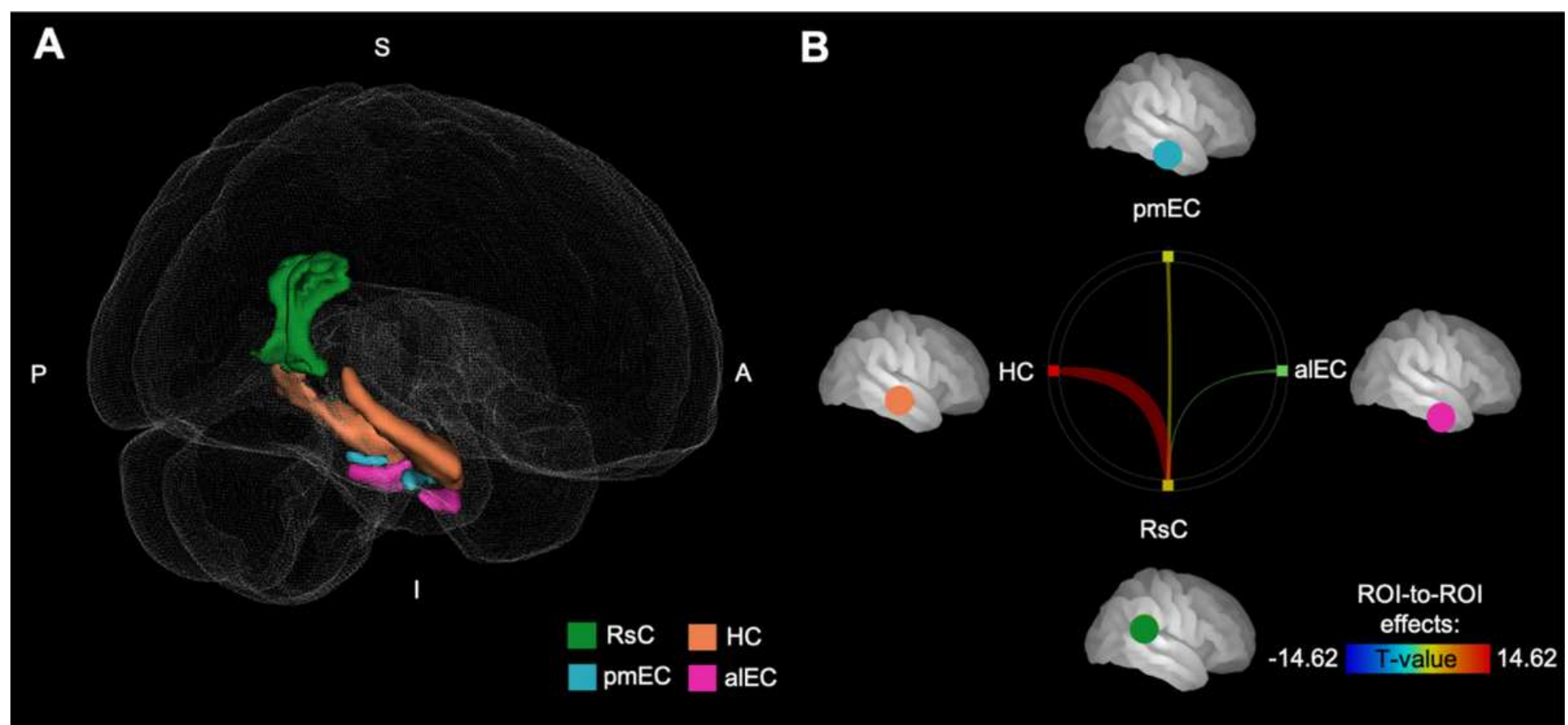

\section{Figure 1}

Hippocampus exhibits strong resting state functional connectivity with retrosplenial cortex. (A) Regions of interest for functional connectivity analysis $(n=97)$. Anterolateral entorhinal cortex (alEC), posteromedial entorhinal cortex (pmEC), and hippocampus $(\mathrm{HC})$ were included from the medial temporal lobe, as well as retrosplenial cortex (RsC). (B) Retrosplenial cortex exhibits functional connectivity with hippocampus $(\beta=0.45, p<0.001)$ and posteromedial entorhinal cortex $(b=0.07, p=0.007)$, but not anterolateral entorhinal cortex $(\beta=0.00, p=0.89)$. Line color and thickness correspond to the T-statistic of semipartial correlations of resting state activity between regions. 


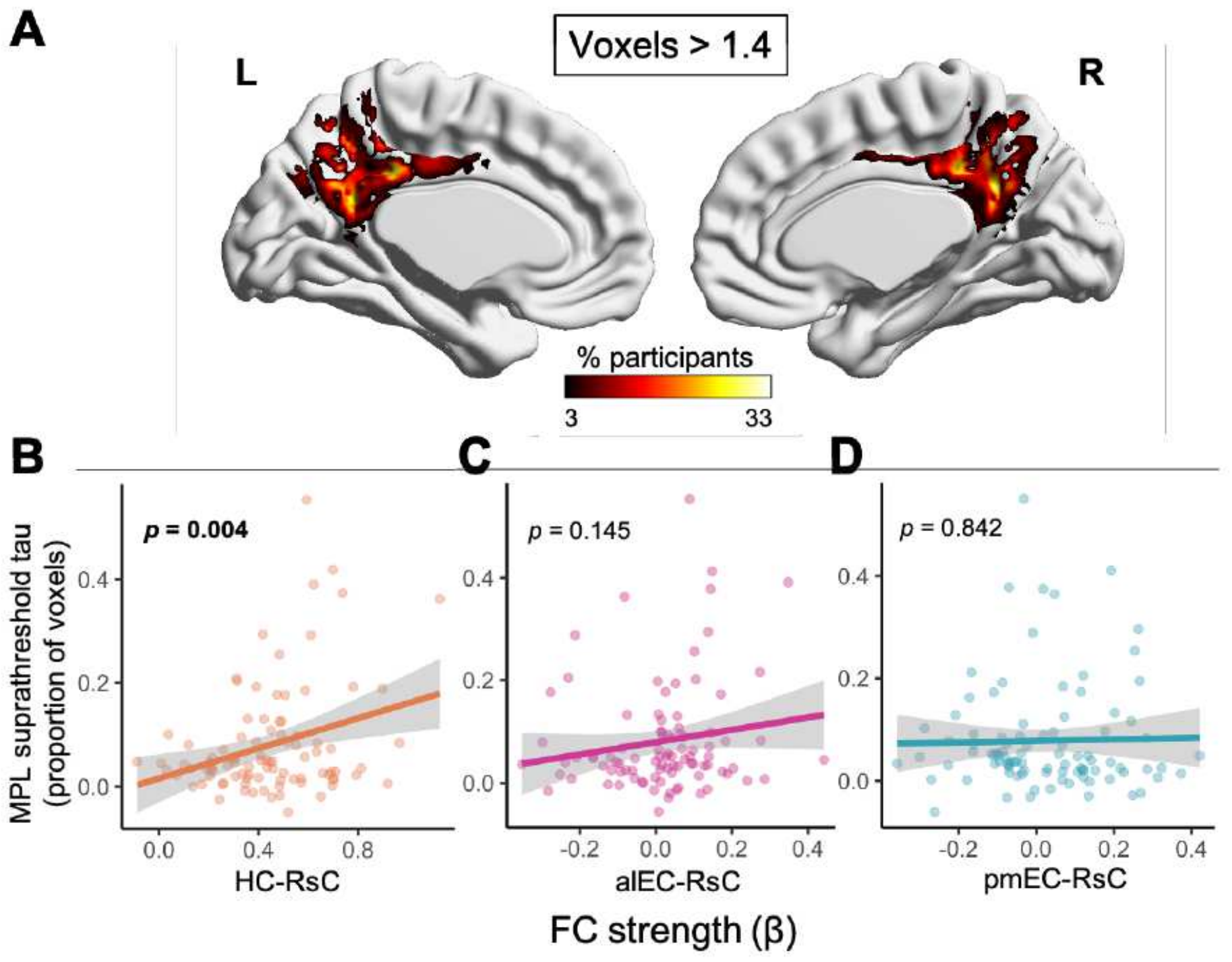

\section{Figure 2}

Hippocampal-retrosplenial connectivity strength is related to medial parietal tau pathology. (A) Percent of participants above tau threshold (FTP SUVR > 1.4) for each voxel in medial parietal lobe (MPL) region comprising retrosplenial cortex, precuneus, and posterior cingulate cortex. (B) Adjusting for age, sex, and global beta amyloid $(A \beta)$, hippocampal-retrosplenial (HC-RsC) resting state functional connectivity strength is associated with mean suprathreshold tau within MPL. MPL suprathreshold tau defined as proportion of voxels above threshold (FTP SUVR > 1.4) within composite region. (C) Anterolateral entorhinal cortex-retrosplenial (alEC-RsC) and (D) posteromedial entorhinal cortex-retrosplenial (pmEC$\mathrm{RsC}$ ) connectivity strength are not associated with MPL tau. Significance value for each plot corresponds to effect of each term from linear regression model. Functional connectivity (FC) strength quantified as bvalues extracted from semipartial correlations between regions. 


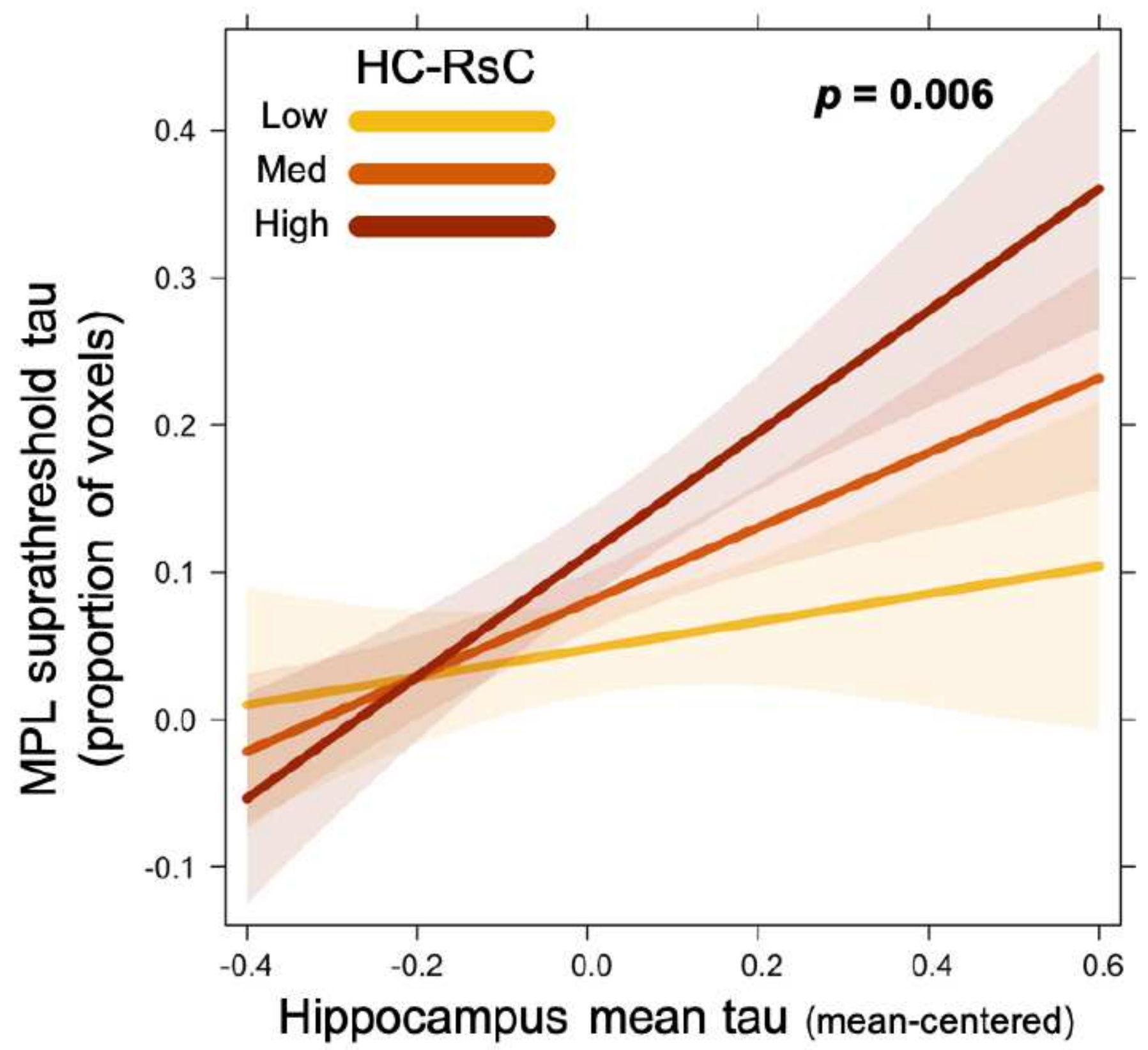

Figure 3

Hippocampal-retrosplenial connectivity modulates the relationship between medial temporal and medial parietal tau. Visualization of interaction between hippocampus mean tau (partial volume corrected FTP SUVR) and hippocampal-retrosplenial functional connectivity strength (HC-RsC) from linear regression model (see Table 2). Medial parietal lobe (MPL) suprathreshold tau is associated with a hippocampal tau $\triangle H C-R s C$ interaction. Plot displays the relationship predicted by linear regression at low (10th percentile), median, and high (90th percentile) HC-RsC. 


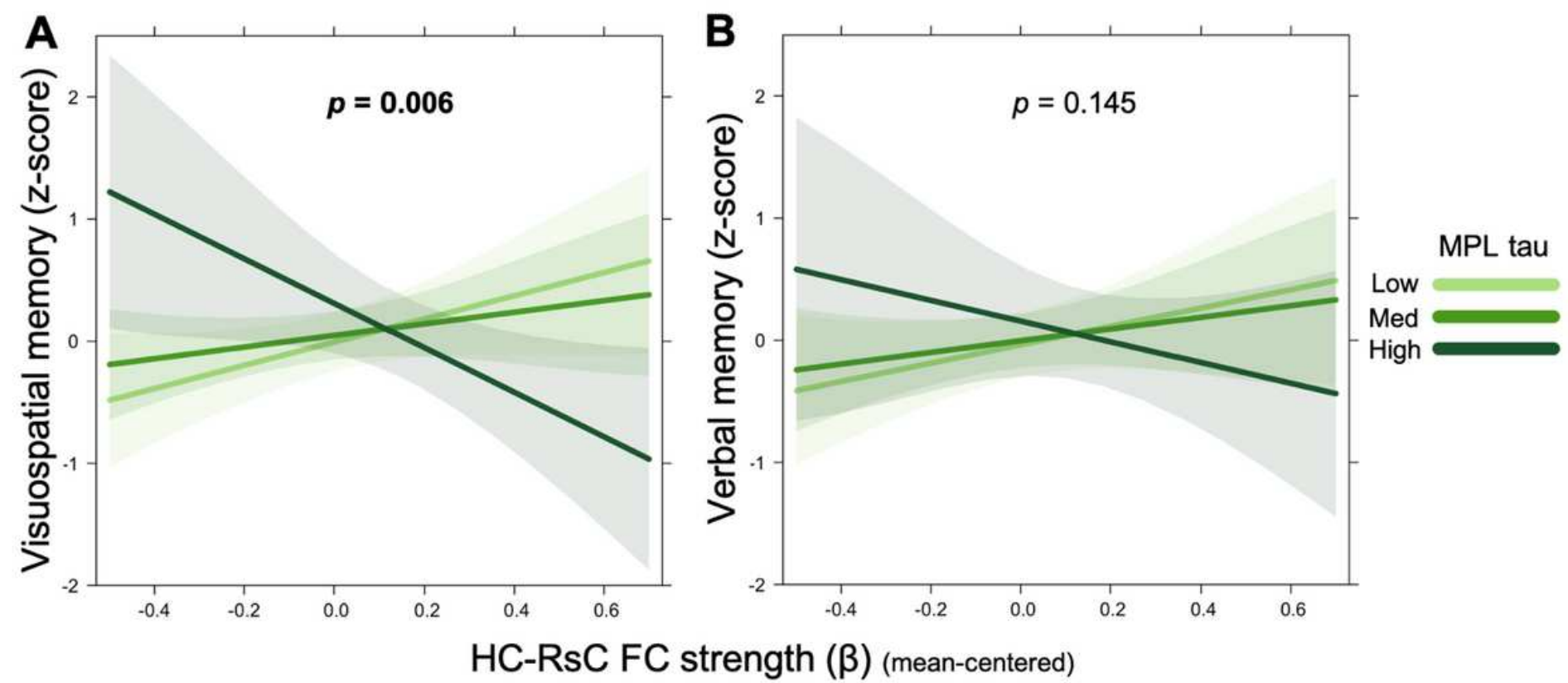

Figure 4

Episodic memory performance is associated with the interaction of hippocampal-retrosplenial connectivity and medial parietal tau. Visualization of interaction between hippocampal-retrosplenial (HCRsC) functional connectivity (FC) strength (beta value) and medial parietal lobe (MPL) suprathreshold tau in relation to visuospatial and verbal memory, adjusting for age, sex, years of education, practice effects, and global A $\mathrm{B}$. (A) Visuospatial memory (Visual Reproduction immediate and delay recall) is associated with the interaction of MPL tau and HC-RsC FC strength. (B) Verbal memory (CVLT immediate and long delay free recall) does not exhibit a significant interaction. Plots display the relationship predicted by linear regression at low (10th percentile), median, and high (90th percentile) MPL suprathreshold tau. 